\title{
Fundamentals of Picosecond Laser Ultrasonics
}

\author{
Osamu Matsuda ${ }^{\mathrm{a}}$, Maria-Cristina Larciprete ${ }^{\mathrm{b}}$, \\ Roberto Li Voti ${ }^{\mathrm{b}}$, Oliver B. Wright ${ }^{\mathrm{a}}$ \\ ${ }^{a}$ Division of Applied Physics, Faculty of Engineering, \\ Hokkaido University, Sapporo 060-8628, Japan \\ ${ }^{b}$ Dipartimento di Scienze di Base ed Applicate per l'Ingegneria, Sapienza Università di Roma, \\ Via A. Scarpa 14, 00161 Roma, Italy
}

\begin{abstract}
The aim of this article is to provide an introduction to picosecond laser ultrasonics, a means by which gigahertz-terahertz ultrasonic waves can be generated and detected by ultrashort light pulses. This method can be used to characterize materials with nanometre spatial resolution. With reference to key experiments, we first review the theoretical background for normalincidence optical detection in opaque single-layer isotropic thin films. The theory is extended to handle isotropic multilayer samples, and again compared to experiment. Then we review applications to anisotropic samples, including oblique-incidence optical probing, and treat the generation and detection of shear waves. Solids including metals and semiconductors are mainly discussed, although liquids are briefly mentioned.

Keywords: acoustics, ultrasonics, optics, gigahertz, terahertz, laser, picosecond, ultrashort, nanometer, nanomaterial, thin film, multilayer, strain, stress, echo, interferometer, reflectance, Green's function, shear waves, longitudinal waves.
\end{abstract}




\section{Introduction}

Picosecond laser ultrasonics, or picosecond ultrasonics, is the study of materials using high frequency acoustic pulses generated and detected by ultrashort optical pulses typically $<1 \mathrm{ps}$ in duration.[1-5] When such an optical pulse, known as a pump pulse, is incident on the surface of a solid, some optical energy is absorbed and converted to heat. In the simplest models one assumes that the process of conversion to heat is instantaneous and occurs within a depth approximately equal to the optical absorption depth. In reality nonequilibrium electron heating and relaxation processes will delay the heat generation, and diffusion processes will spatially blur the excitation region. The production of heat in a solid results in a lattice temperature rise, and this leads to a thermal stress that can propagate in three dimensions. We shall concentrate on the acoustic propagation normal to the surface, because it is in this direction in an isotropic solid that the frequency of the generated acoustic pulse is highest and the wavelength smallest. If the optical spot size (typically a few microns) is much larger than the optical absorption depth ( 10-50 nm for example), the generated acoustic pulse can be accurately modelled as a superposition of longitudinal plane waves travelling normal to the surface. Acoustic frequencies are usually in the $10-1000 \mathrm{GHz}$ range. Because of the correspondingly small acoustic wavelength, down to the nanometer range, picosecond laser ultrasonics is ideal for investigating thin films and nanostructures.

Sub-surface structures or inhomogeneous regions under the surface, typically at $\mathrm{nm}$ to $\mu \mathrm{m}$ depths, can reflect the acoustic pulse back to the surface. This is shown schematically in Fig. 1 for the example of an opaque thin film on a substrate. We can detect the return of the acoustic pulse to the surface by illuminating the sample with a second ultrashort light pulse, known as a probe pulse, focused to the same point on the sample. A set of measurements of the probe beam reflectivity or phase change is obtained by changing the time delay between the pump and probe pulses in an optical delay line, thus avoiding the need for an ultrafast photodetector. This is known as the optical pump and probe technique. Noise reduction is achieved by chopping the pump beam and using lock-in detection. We shall deal separately with the acoustic generation and detection processes without going into any details of the experimental apparatus.

The purpose of this article is to provide a summary of the fundamentals of generation and detection of longitudinal acoustic pulses in picosecond laser ultrasonics. It should serve as an introduction to the subject for those interested in this field. After considering the theory of acoustic generation through the thermoelastic effect in an opaque isotropic solid in the absence of diffusion processes, we briefly review the effect of electron and thermal diffusion processes and other generation mechanisms on the generated acoustic pulses. We then present the theory of optical detection using a normally-incident probe beam, including the detection of both optical reflectivity and phase changes. Different contributions to the acoustic echoes are explained and elucidated using the example of thermoelastically generated acoustic pulses in the absence of diffusion processes. Finally, advances in oblique-incidence detection techniques and the generation of picosecond shear waves are briefly reviewed. 

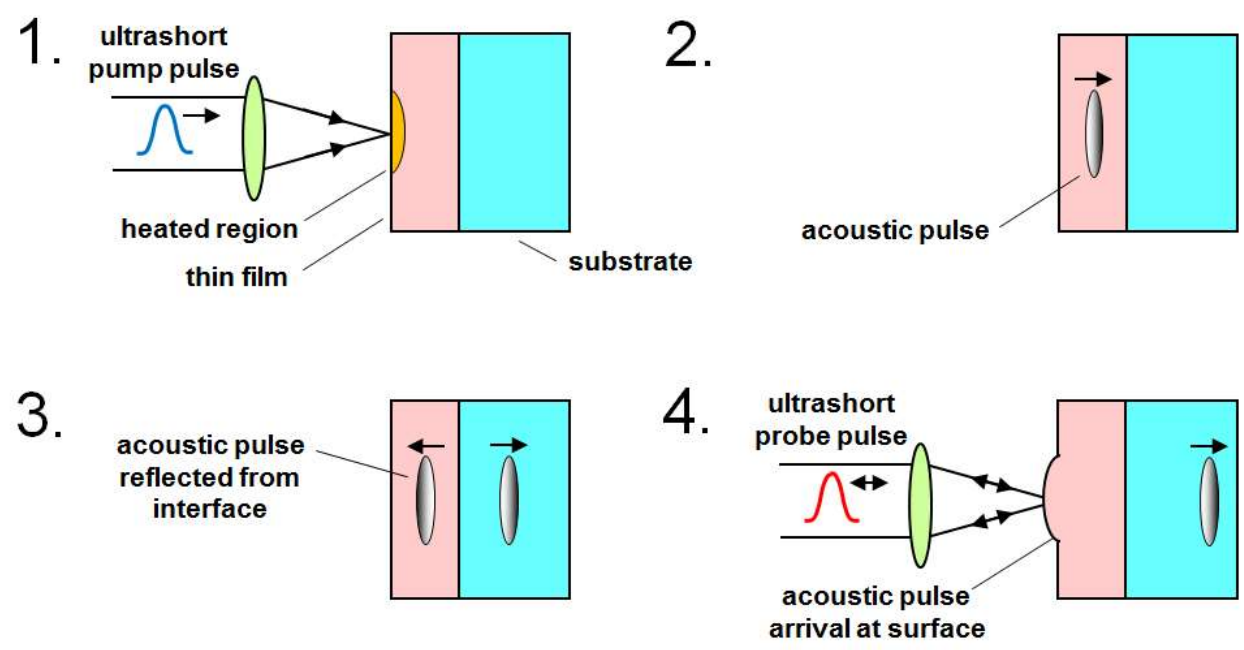

Fig. 1: Sequence of events in picosecond laser ultrasonics.

\section{Basic theory of laser picosecond ultrasonics}

\subsection{Theory of picosecond strain generation and propagation}

\section{Thermal stress}

When an ultrashort optical pulse is absorbed at a free surface it produces a thermal stress. To calculate the strain pulse shape we first need to know the effect of thermal stress on the stress-strain relation.[6] We shall assume an infinitely wide illumination of a solid by an infinitely short optical pulse. This is a one-dimensional problem in which acoustic diffraction effects do not occur. We shall also at first neglect the effect of any electron or thermal diffusion processes. The thermal expansion tensor $\alpha_{i j}$ of a solid (expressed as a $3 \times 3$ matrix) is defined by $\eta_{i j}=\alpha_{i j} \Delta T$, where $\eta_{i j}$ is the strain tensor, here describing a mechanical equilibrium situation, and $\Delta T$ is the position-dependent change in temperature compared to a reference temperature before the optical pulse arrival. For small displacements, the strain tensor is given by $\eta_{i j}=\left(\partial u_{i} / \partial x_{j}+\right.$ $\left.\partial u_{j} / \partial x_{i}\right) / 2$, where $u_{i}$ is the displacement vector and $x_{i}$ is the position vector. If a material undergoes an instantaneous change of temperature at time $t=0$, it will expand at a rate governed by the speed of sound. The relation between the stress tensor $\sigma_{i j}$ and strain $\eta_{i j}$ in the presence of a thermal stress $\sigma_{i j}{ }^{\prime}$ is given by[7]

$$
\sigma_{i j}=c_{i j k l}+\sigma_{i j}{ }^{\prime}
$$

where $c_{i j k l}$ is the elastic constant tensor, and the summation is understood to be over repeated subscripts. When the solid has expanded (at time $t=\infty$ in the case of no thermal diffusion), $\sigma_{i j}=0$ and $\eta_{i j}=\alpha_{i j} \Delta T$. So, from Eq. (1), $\sigma_{i j}{ }^{\prime}=-c_{i j k l} \alpha_{k l} \Delta T$, where $\Delta T$ is a function of $x, y$, and $z$ in general. For isotropic solids, we define

$$
\sigma_{i j}=2 \mu \eta_{i j}+\lambda \eta_{k k} \delta_{i j}
$$

where $\lambda$ and $\mu$ are elastic constants known as the Lamé constants, and $\delta_{i j}=1$ when 
$i=j$ but $\delta_{i j}=0$ otherwise. So

$$
\sigma_{i j}{ }^{\prime}=-\left(2 \mu \alpha_{i j}+\lambda \alpha_{k k} \delta_{i j}\right) \Delta T
$$

This can be seen by comparison with Eq. (2). Alternatively, Eq. (2) implies that $c_{i j k l}=$ $2 \mu \delta_{i k} \delta_{j l}+\lambda \delta_{i j} \delta_{k l}$. However, for isotropic solids, $\alpha_{i j}=\alpha \delta_{i j}$, where $\alpha$ is the coefficient of linear thermal expansion. So

$$
\sigma_{i j}{ }^{\prime}=-(2 \mu+3 \lambda) \alpha \delta_{i j} \Delta T
$$

Alternatively, in terms of the bulk modulus $B$, Poisson's ratio $v$, and Young's modulus $Y$, where the definitions

$$
B=\frac{Y}{3(1-2 v)}, v=\frac{\lambda}{2(\lambda+\mu)}, Y=\frac{\mu(2 \mu+3 \lambda)}{(\lambda+\mu)}
$$

apply, we may write $(2 \mu+3 \lambda)=3 B$. Therefore, for isotropic solids,

$$
\sigma_{i j}{ }^{\prime}=-3 B \alpha \delta_{i j} \Delta T
$$

The generated thermal stress is hydrostatic and compressive for positive $\alpha$ and $\Delta T$.

\section{Generation of longitudinal waves with picosecond optical pulses in isotropic materials}

We assume that $\Delta T=\Delta T(z, t)$ is a function of depth $z$ and time $t$ only, where $z=0$ corresponds to the flat sample surface and $+z$ is directed into the material. $\Delta T(z, t)$ is assumed to be zero for $t<0$, and $\Delta T=\Delta T(z)$ to be independent of time for $t>0$ (in the absence of thermal diffusion). Also, because of this one-dimensional model, the only non-zero tensor component of the strain is $\eta_{z z}$. For an isotropic solid,

$$
\sigma_{z z}=(2 \mu+\lambda) \eta_{z z}-3 B \alpha \Delta T(z)
$$

Also, $\sigma_{x x}=\sigma_{y y}=\lambda \eta_{z z}-3 B \alpha \Delta T(z)$. The components $\sigma_{x x}$ and $\sigma_{y y}$ are present to prevent lateral contraction (i.e., to make $\eta_{x x}=\eta_{y y}=0$ ). Equation (3) can be rewritten as

$$
\sigma_{z z}=3 \frac{1-v}{1+v} B \eta_{z z}-3 B \alpha \Delta T(z)=\rho_{0} v_{l}^{2} \eta_{z z}-3 B \alpha \Delta T(z)
$$

where $v_{l}$ is the longitudinal sound velocity and $\rho_{0}$ is the density. The elastic wave equation for zero body forces, expressed in terms of the $z$-directed displacement $u_{z}$, where $\eta_{z z}=\partial u_{z} / \partial z$, is given by

$$
\begin{aligned}
& \frac{\partial \sigma_{z z}}{\partial z}=\rho_{0} \frac{\partial^{2} u_{z}}{\partial t^{2}}, \\
& u_{z}(z, t)=\int_{+\infty}^{z} \eta_{z z}\left(z^{\prime}, t\right) d z^{\prime} .
\end{aligned}
$$

To solve the elastic wave equation we need to know the form of $\Delta T(z)$. For 
ultrashort pulse optical absorption at the surface of a homogeneous isotropic solid,

$$
\Delta T(z)=\frac{(1-R) Q}{A C \zeta_{0}} e^{-\frac{z}{\zeta_{0}}} \text { for } t>0, \Delta T(z, t)=0 \text { for } t<0,
$$

where $R$ is the optical intensity reflection coefficient, $Q$ is the incident optical pulse energy, $A$ is the area over which the energy $Q$ is distributed (assumed to be a uniform distribution), $C$ is the heat capacity per unit volume, and $\zeta_{0}$ is the optical absorption depth of the pump light.

For the boundary condition, there should be no stretching perpendicular to the surface at the surface itself, since there cannot be any restoring force there. Therefore, $\sigma_{z z}=0$ at $z=0$. From Eq. (4) this means that at any time $t>0$, for $z=0$ the relation $\eta_{z z}=\eta_{0}$ should hold, where

$$
\eta_{0}=\frac{3 B \alpha(1-R) Q}{A C \zeta_{0} \rho_{0} v^{2}}
$$

For the initial conditions, $\eta_{z z}=0, \partial \eta_{z z} / \partial t=0$, and $u_{z}=0$ for all $z$ for $t<0$. Using Eqs. (4)-(6), the wave equation for $u_{z}$ can be written as

$$
\frac{\partial^{2} u_{z}}{\partial t^{2}}=v_{l}^{2} \frac{\partial^{2} u_{z}}{\partial z^{2}}+\frac{v_{l}^{2} \eta_{0}}{\zeta_{0}} e^{-\frac{z}{\zeta_{0}}}
$$

The general solution is given by

$$
u_{z}=f\left(z-v_{l} t\right)+g\left(z+v_{l} t\right)-\zeta_{0} \eta_{0} e^{-\frac{z}{\zeta_{0}}}
$$

In order to find $f$ and $g$, we extend the functions $f$ and $g$ to apply also to the $z<$ 0 region in such a way as to satisfy the boundary condition. This can be done by assuming that we have an even function for displacement at all times. This is equivalent to converting our problem into a more symmetrical form by the addition of an imaginary identical material in front of the surface. For $z>0$,

$$
u_{z}=f\left(z-v_{l} t\right)+g\left(z+v_{l} t\right)-\zeta_{0} \eta_{0} e^{-\frac{z}{\zeta_{0}}}
$$

and, for $z<0$ (in front of the material surface),

$$
\begin{gathered}
u_{z}=f\left(|z|-v_{l} t\right)+g\left(|z|+v_{l} t\right)-\zeta_{0} \eta_{0} e^{-\frac{|z|}{\zeta_{0}}} \\
=f\left(-z-v_{l} t\right)+g\left(-z+v_{l} t\right)-\zeta_{0} \eta_{0} e^{\frac{z}{\zeta_{0}}} .
\end{gathered}
$$

To be consistent (i.e., to have a single solution for all positive and negative $z$ ) the functions representing propagation in the same direction should be chosen to be identical. (Because the waves travelling in the same direction join up smoothly at $z=0$ as there is no longer an interface there in this new problem). So $f\left(z-v_{l} t\right)=$ $g\left(-z+v_{l} t\right)$ and $g\left(z+v_{l} t\right)=f\left(-z-v_{l} t\right)$ for all $z$ and $t$ from the above two equations. Therefore

$$
u_{z}=f\left(z-v_{l} t\right)+f\left(-z-v_{l} t\right)-\zeta_{0} \eta_{0} e^{-\frac{|z|}{\zeta_{0}}}(\text { valid for } z>0 \text { or } z<0)
$$


and

$$
\frac{\partial u_{z}}{\partial z}=f^{\prime}\left(z-v_{l} t\right)-f^{\prime}\left(-z-v_{l} t\right)+\eta_{0} e^{-\frac{|z|}{\zeta_{0}}} \operatorname{sgn}(z)
$$

where $\operatorname{sgn}(z)=1$ for $z>0,-1$ for $z<0,0$ for $z=0$.

Now we can use the initial condition $\partial u_{z} /\left.\partial z\right|_{t=0}=0$ : from the above equation, $f^{\prime}(z)-f^{\prime}(-z)=-\eta_{0} e^{-\frac{|z|}{\zeta_{0}}} \operatorname{sgn}(z)$. In general the equation $F(z)-F(-z)=G(z)$, where $G(z)$ is an odd function, can be solved uniquely with $F(z)=-F(-z)=$ $G(z) / 2$ :

$$
f^{\prime}(z)=-\frac{\eta_{0}}{2} e^{-\frac{|z|}{\zeta_{0}}} \operatorname{sgn}(z)
$$

With this choice of $f^{\prime}(z)$ the boundary condition, $\left.\eta_{z z}\right|_{z=0}=\partial u_{z} /\left.\partial z\right|_{z=0}=\eta_{0}$, is automatically satisfied. (It should be because we chose to extend the problem to both positive and negative values of $z$ in order to satisfy the boundary condition.)

Combining equations we obtain, for all $z$ :

$$
\eta_{z z}=\eta_{0} e^{-\frac{z}{\zeta_{0}}} \operatorname{sgn}(z)+\frac{\eta_{0}}{2}\left[e^{-\frac{\left|z+v_{l} t\right|}{\zeta_{0}}} \operatorname{sgn}\left(-z-v_{l} t\right)-e^{-\frac{\left|z-v_{l} t\right|}{\zeta_{0}}} \operatorname{sgn}(z-\right.
$$

$\left.\left.v_{l} t\right)\right]$

Unlike the displacement $u_{z}$, this is an odd function of $z$. We only require the solution for $z>0:[1,8]$

$$
\eta_{z z}=\eta_{0} e^{-\frac{z}{\zeta_{0}}}-\frac{\eta_{0}}{2}\left[e^{-\frac{z+v_{l} t}{\zeta_{0}}}+e^{-\frac{\left|z-v_{l} t\right|}{\zeta_{0}}} \operatorname{sgn}\left(z-v_{l} t\right)\right] \text {. }
$$

The strain consists of a constant first term and a propagating second term. The reflection coefficient for the strain at the surface is -1 . Because of this, the propagating component is bipolar, as shown in Fig. 2 (a). It consists of two decaying exponentials of decay length $\zeta_{0}$ stitched together with opposite signs. The time it takes for the strain to reach a significant strength is $\sim \zeta_{0} / v_{l}$, the sound propagation time across $\zeta_{0}$. After the propagating strain component has left the near-surface region, corresponding to a time $\gtrsim 5 \zeta_{0} / v_{l}$ [see Fig. 2 (a)], a constant and spatially exponentially decaying strain is evident near the surface owing to the thermal expansion there.

The wavelength of this strain pulse is of the order of $2 \pi \zeta_{0}$. (The frequency of the maximum strain amplitude is $f=v_{l} /\left(2 \pi \zeta_{0}\right)$, as explained below.) To find the stress $\sigma_{z z}$ we substitute for $\eta_{z z}$ in Eq. (4). For $z>0$,

$$
\sigma_{z z}=-\frac{\rho_{0} v_{l}^{2} \eta_{0}}{2}\left[e^{-\frac{z+v_{l} t}{\zeta_{0}}}+e^{-\frac{\left|z-v_{l} t\right|}{\zeta_{0}}} \operatorname{sgn}\left(z-v_{l} t\right)\right] \text {. }
$$

In contrast to the strain, there is no stress left near the surface of the solid after the stress pulse has had time to propagate away from the surface (i.e. a long way compared to $\zeta_{0}$ ). 
Otherwise the form of the stress and strain is identical. Just after the arrival of the optical pulse the longitudinal stress is. $\sigma_{z z}(z, 0)=-\rho_{0} v_{l}^{2} \eta_{0} \exp \left(-\mathrm{z} / \zeta_{0}\right)$. If $\alpha>0$ and $\quad \Delta T>0$ this stress is compressive, and so is the leading edge of the strain pulse.

If we take the origin of the spatial coordinates at the centre of the strain pulse when the strain is far from the sample surface, then the temporal Fourier transform of this propagating strain component is given by

$$
\int_{-\infty}^{\infty} \eta_{z z}(z, t) e^{i \omega t} d t=\eta_{0} \frac{i \omega}{\omega^{2}+1 / \tau^{2}},
$$

where $\omega$ is the acoustic angular frequency and $\tau=\zeta_{0} / v_{l}$ is the sound propagation time across the optical absorption depth $\zeta_{0}$. For $\zeta_{0}=10 \mathrm{~nm}$ and $v_{l}=5 \mathrm{~km} \mathrm{~s}^{-1}$, as is typical for metals, the modulus of the frequency spectrum has a peak at $f=$ $v_{l} /\left(2 \pi \zeta_{0}\right) \sim 80 \mathrm{GHz}$, corresponding to an acoustic wavelength of about $60 \mathrm{~nm}$. Figure 2 (b) shows the calculated modulus of the ultrasonic frequency spectrum for chromium using blue light, in which case $v_{l}=6.65 \mathrm{~km} \mathrm{~s}^{-1}, \zeta_{0}=13.5 \mathrm{~nm}$ and $f=$ $v_{l} /\left(2 \pi \zeta_{0}\right) \sim 80 \mathrm{GHz} .[9]$

The more general problem of thermoelastic strain generation at the surface of opaque anisotropic medium has been discussed in terms of temporal Fourier transforms and spatial Laplace transforms including the residue theorem.[10]
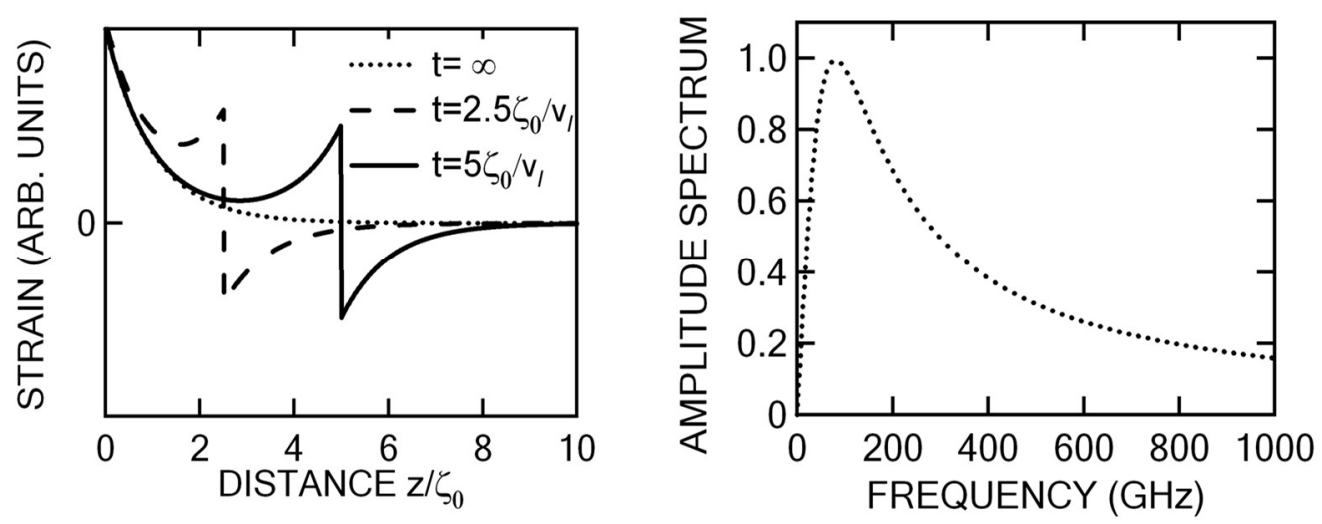

Fig. 2: (a) Plot of the longitudinal strain $\eta_{z z}(z)$ in an isotropic solid of optical absorption length $\zeta_{0}$ and longitudinal sound velocity $v_{l}$ at different times after the arrival at $t=0$ of an ultrashort optical pulse at the surface (for $\eta_{0}>0$ ). (b) Plot of the calculated normalized modulus of the frequency spectrum of an acoustic pulse excited in chromium by an ultrashort blue light pulse of wavelength $415 \mathrm{~nm}$ with $\zeta_{0}=13.5 \mathrm{~nm}$. The effects of diffusion are ignored in the calculation.

\section{Diffusion Process and Other Strain Generation Mechanisms}

If diffusion processes are taken into account, the generated strain pulse is broadened for two reasons: (i) the generation process is spread out over time; (ii) the generation process is spread out over space. In metals one needs to take into account the effects of both thermal and electron diffusion.[1, 2, 5, 9-13] Figure 3 shows the effect of including diffusion processes in the calculation of the strain pulse shape for chromium when pumped with ultrashort light pulses at a central wavelength of $415 \mathrm{~nm}$.[9] The result of including both diffusion processes is a broadened strain pulse with a peak in the 
frequency spectrum reduced to $50 \mathrm{GHz}$.

In semiconductors one needs to take into account both thermal and carrier diffusion.[1, 14-16]. Moreover, the strain produced in semiconductors by excitation with an ultrashort light pulse depends strongly on the carrier density as well as on the temperature change. In this case, Eq. (4) must be modified to include the electronic stress contribution arising from the excited carriers, because, in general, the carrier lifetime will not be negligible compared to the acoustic pulse durations involved:[1]

$$
\sigma_{z z}=\rho_{0} v_{l}^{2} \eta_{z z}-3 B \alpha \Delta T(z)+\sum_{\mathbf{k}} \frac{\partial E_{\mathbf{k}}}{\partial \eta_{z z}} N_{\mathbf{k}}
$$

where $\partial E_{\mathbf{k}} / \partial \eta_{z z}$ is the deformation potential and $N_{\mathbf{k}}$ is the carrier density for a carrier of energy $E_{\mathbf{k}}$ and wave vector $\mathbf{k}$. This extra term can be understood through arguments based on statistical mechanics, and it is often a good approximation to replace it by $-B N \partial E_{g} / \partial p$, where $E_{g}$ is the band gap and $N$ is the total excited carrier density. (This is analogous to the formula for the pressure $p=\sum_{i} p_{i}\left(-d E_{i} / d V\right)$ according to the canonical distribution for a series of energy levels $E_{i}$ with occupation probability $p_{i}$ for a system of volume $V$.) In the case of GaAs (100) excited at a wavelength of 375 $\mathrm{nm}$, for example, the strain generated by the electronic stress is 6 times larger than the strain generated by the thermoelastic stress, but of the same sign.[11] In experiments on Si (100) a dominant strain was generated by the electronic stress and was observed to have the opposite sign to the thermal expansion, as evidenced by a hollow appearing in the surface on irradiation with ultrashort light pulses of wavelength $630 \mathrm{~nm}$.[17]

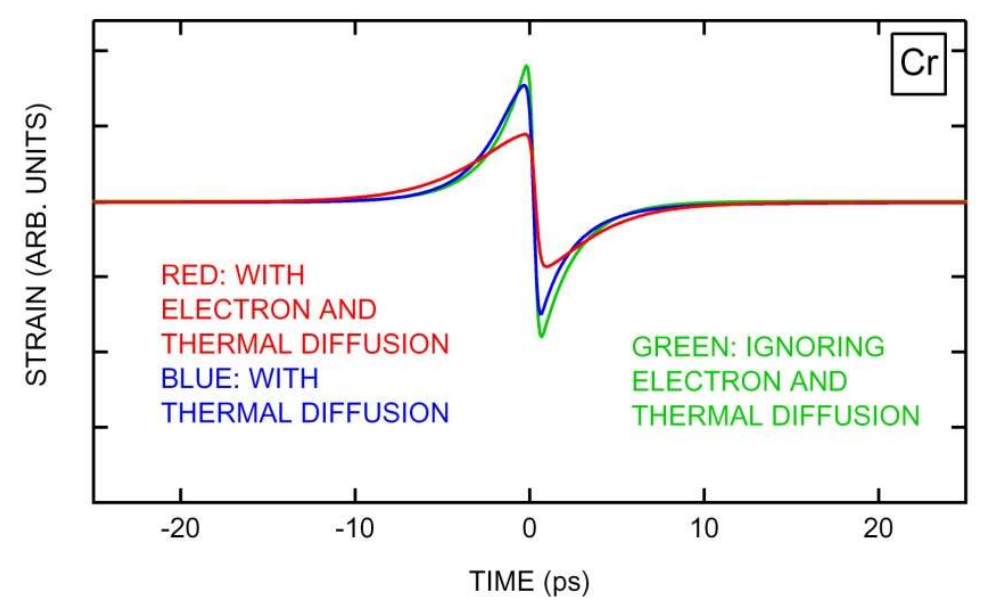

Fig. 3: Plot of the calculated strain as a function of time for chromium when excited with ultrashort light pulses at $415 \mathrm{~nm}$ wavelength, showing the effect of diffusion processes. Green: no diffusion, blue: with thermal diffusion only, red: including both thermal and electron diffusion.

In piezoelectric materials there is a strong contribution from the generation of timedependent electric fields. This contribution may completely dominate the generated strain and observed optical reflectance changes. [18-23]

\section{Acoustic generation in more complicated sample structures}

In multilayer or more complicated sample geometries, the optical absorption characteristics of the sample need to be worked out to calculate the generated strain. $[8$, 
24] As with single-layer samples this will in general depend on the wavelength, angle of incidence, state of polarization and pulse duration of the optical beam. In addition, any diffusion effects need to be taken into account to determine the time dependent strain distribution. If the symmetry of the strain generation is broken by crystal anisotropy or by complicated three-dimensional sample geometries, it may be necessary to solve the relevant equations by numerical methods and to consider the excitation of both longitudinal and shear acoustic waves.[21,25]

The sample may also contain elements that are transparent. One particular example is a sample consisting of a thin transparent film on an opaque substrate. Another very similar example is a thick opaque metal film on a transparent substrate with the optical illumination from the transparent substrate side. Instead of the bipolar strain pulse shape one obtains a unipolar pulse shape in the transparent material. The strain pulse shape in the opaque material can be either unipolar or bipolar, depending on acoustic impedances $\left(\begin{array}{ll}\rho_{0} & v_{l}\end{array}\right)$ of the materials used.[26, 27] The growth of transparent thin films can be monitored by picosecond laser ultrasonics, as was shown for the case of ice films.[28]

In semiconductor superlattices or heterostructures, or other partially transparent multilayers, optical transfer matrix methods can be used to determine the generated strain distribution, although this may rely on the assumption of effective optical constants in very thin layers and can also depend on the shape of the carrier wavefunctions.[16, 18-20, 22, 29-37]

\subsection{Basic theory of optical detection of picosecond strain pulses}

\section{Absorption in isotropic solids}

Consider the case of optical incidence on a sample with an optical probe pulse. First consider a linear, homogeneous, isotropic and lossless medium, for which $\mathbf{D}=\varepsilon \varepsilon_{0} \mathbf{E}$, $\mathbf{B}=\mu_{0} \mathbf{H}$ and $\mathbf{P}=\varepsilon_{0} \chi \quad \mathbf{E}=\varepsilon_{0}(\varepsilon-1) \mathbf{E}$, where $\mathbf{P}, \mathbf{E}$ and $\mathbf{D}$ are the polarization, electric field and electric displacement vectors, $\mathbf{B}$ and $\mathbf{H}$ are the magnetic flux density and the magnetic field strength vectors, and $\varepsilon=1+\chi$ is the relative permittivity, $\varepsilon_{0}$ is the permittivity of free space, $\chi$ is the electric susceptibility, and $\mu_{0}$ is the permeability of free space. The Maxwell's equations are

$$
\begin{aligned}
& \nabla \cdot \mathbf{E}=0, \\
& \nabla \cdot \mathbf{H}=0, \\
& \nabla \times \mathbf{E}=-\mu_{0} \partial \mathbf{H} / \partial t, \\
& \nabla \times \mathbf{H}=\varepsilon \varepsilon_{0} \partial \mathbf{E} / \partial t .
\end{aligned}
$$

We find $\nabla^{2} \mathbf{E}=\left(1 / v^{2}\right) \partial^{2} \mathbf{E} / \partial t^{2}$, and similarly for $\mathbf{H}$. All components of $\mathbf{E}$ and $\mathbf{H}$ obey the same wave equation. Here the optical propagation velocity is $v=$ $1 /\left(\varepsilon \varepsilon_{0} \mu_{0}\right)^{1 / 2}=c / n$, where $n=\varepsilon^{1 / 2}$ is the refractive index of the medium, and $c$ is the speed of light in vacuum. For sinusoidally varying fields that are monochromatic, $\mathbf{E}$, $\mathbf{H} \propto e^{-i \omega t}$, where $\omega$ is the optical angular frequency. So, from the wave equation above,

$$
\nabla^{2} U+k_{1}^{2} U=0
$$

where $U$ is any component of $\mathbf{E}$ or $\mathbf{H}$ and $k_{1}=\omega / v=\omega n / c=k n, k$ being the free-space wave number. The refractive index $n$ is in general a frequency dependent 
quantity.

We can include the absorption of electromagnetic waves if $\chi=\chi^{\prime}+i \chi^{\prime \prime}$ :

$$
k_{1}=\omega\left(\varepsilon \varepsilon_{0} \mu_{0}\right)^{1 / 2}=k(1+\chi)^{1 / 2}=k\left(1+\chi^{\prime}+i \chi^{\prime \prime}\right)^{1 / 2}=k \tilde{n},
$$

where $\tilde{n}=\sqrt{\varepsilon}=\tilde{n}(\omega)=n+i \kappa$ is the complex refractive index. For a plane wave, $\left.U \propto e^{i\left(k_{1} z-\omega t\right)}=e^{i[k(n+i \kappa) z-\omega t]}=e^{i(k n z-}\right) e^{-k \kappa z}$. Energy absorption depends on $U^{2} \propto e^{-2 k \kappa z}=e^{-z / \zeta}$. So $\zeta=1 /(2 k \kappa)=\lambda /(4 \pi \kappa)$, where $\zeta$ is here the optical absorption depth for the probe light.

Reflectance change for a wave reflected from a medium with a perturbed refractive index profile

This section follows and extends the method described by Thomsen et al.[1] First consider the case of normal incidence of a plane-polarized, monochromatic electromagnetic wave on a plane surface of a lossy medium, as summarized in Fig. 4. The stipulation of a monochromatic beam is at odds with the use of an ultrashort light pulse, but it is generally a good assumption for the $>100 \mathrm{fs}$ optical pulse durations typically used to ignore the slight spread $(<20 \mathrm{~nm})$ in wavelength at visible or nearvisible wavelengths. We assume that $\mathbf{E}$ is polarized in the $x$ direction, where $z$ is the coordinate in the depth direction. The boundary conditions for $\mathbf{E}$ and $\mathbf{H}$ (i.e., parallel components continuous) and the Maxwell equation, $\partial H_{y} / \partial z=i \omega \varepsilon \varepsilon_{0} E_{x}$, lead to the standard Fresnel equations shown in Fig. 4 for the reflectance $r_{0}$ and transmittance $t_{0}$ at normal incidence. (These will be in fact derived later on in Section 3.)

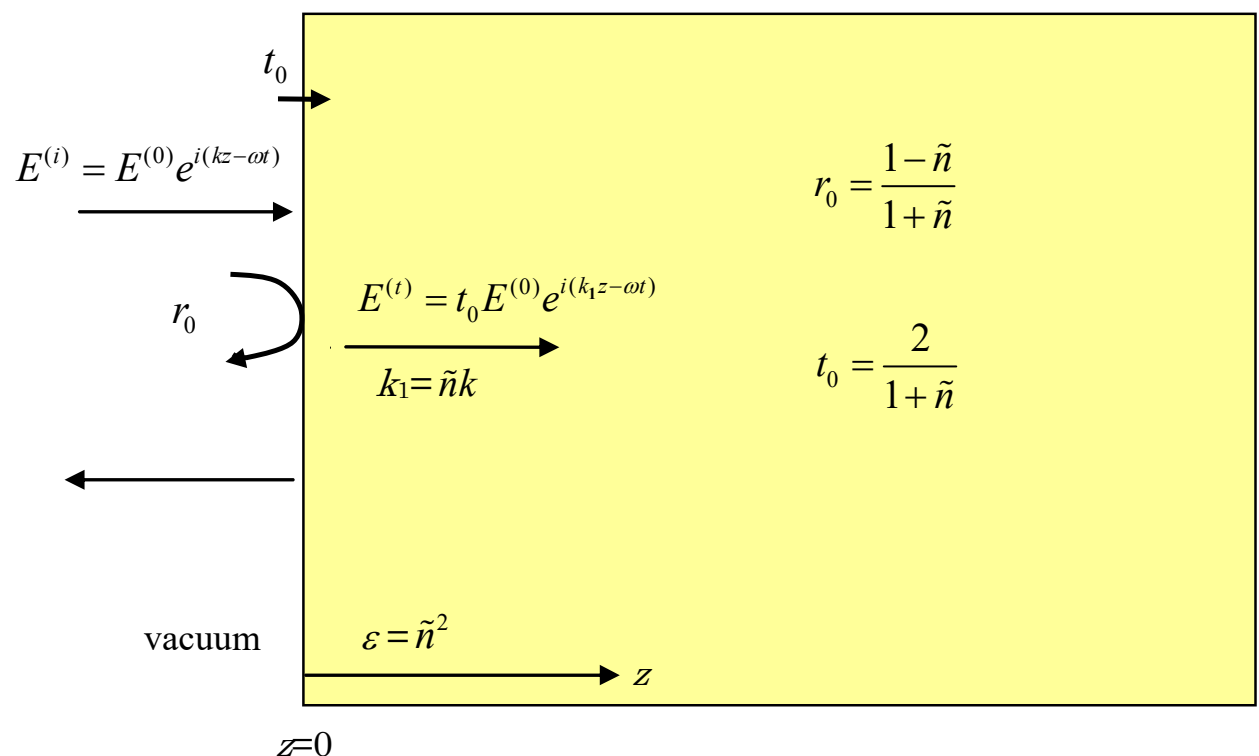

Fig. 4: Diagram to show the electric fields for a plane linearly-polarized monochromatic electromagnetic wave normally incident on a linear lossy medium.

Now consider the case of a small, $z$-dependent variation in permittivity $\Delta \varepsilon(z) \ll \varepsilon$. In addition to arising from the presence of propagating and static strain, in general this modulation can be caused by the diffusion and relaxation of nonequilibrium electron 
and hole distributions or by thermal diffusion. Here, however, we restrict our attention to perturbations involving (propagating) strain. The wave equation for normal optical incidence becomes

$$
\frac{\partial^{2} E_{x}(z)}{\partial z^{2}}=-k^{2}[\varepsilon+\Delta \varepsilon(z)] E_{x}(z)
$$

Let us first solve this equation for the case of a spatial $\delta$-function variation in permittivity: $\Delta \varepsilon(z)=F \delta\left(z-z^{\prime}\right)$. This corresponds to the strain pulse being at a depth $z^{\prime}$ below the sample surface, as shown in Fig. 5. In the figure the electric field incident (inside the medium) on the strain pulse is defined so that $E_{i}=E_{0}$ and $t_{0} E^{(0)}=$ $E_{0} e^{-i k_{1} z^{\prime}}$, allowing the boundary conditions at $z=z^{\prime}$ to be applied with ease.

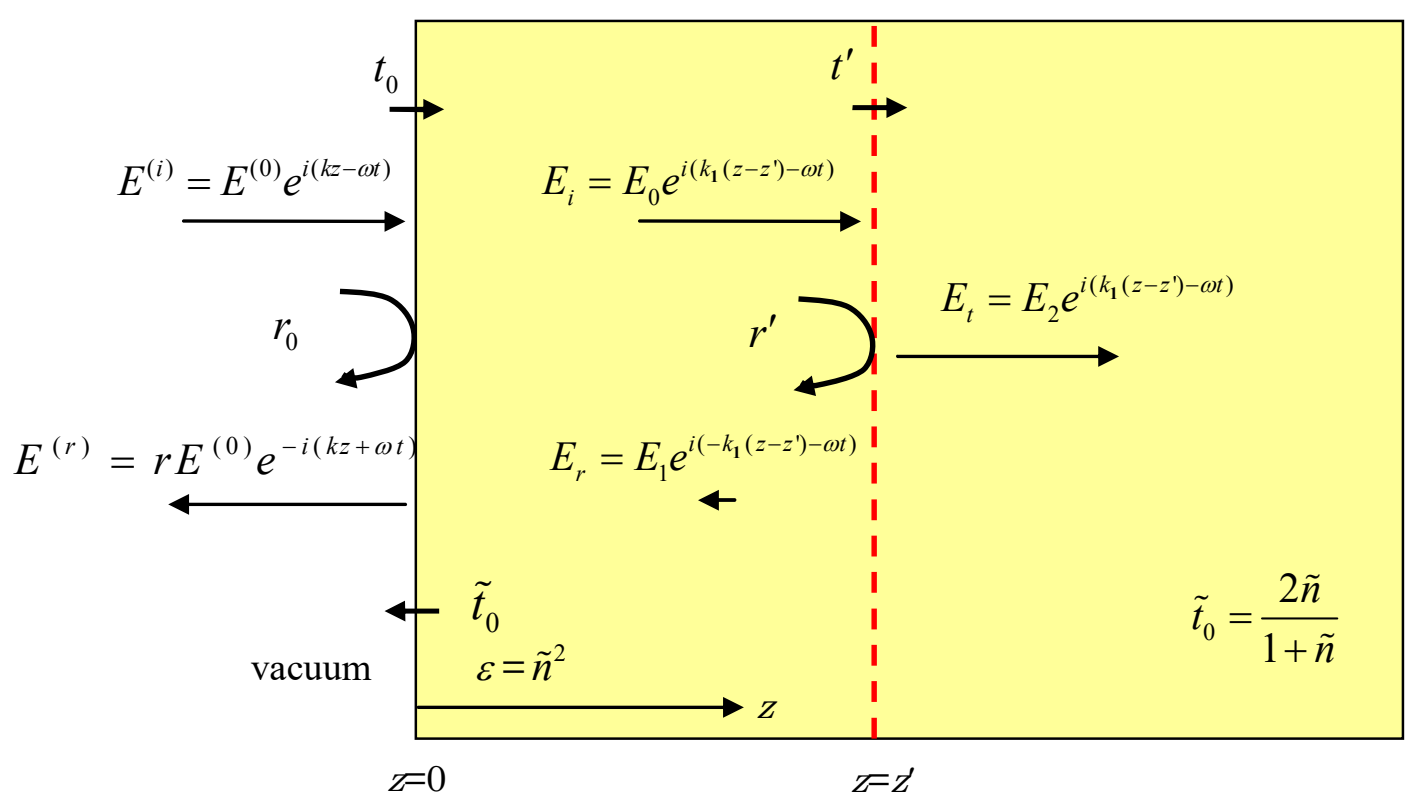

Fig. 5: Diagram to show the electric fields for a plane linearly-polarized monochromatic electromagnetic wave normally incident on a linear lossy medium containing a strain pulse at position $z=z^{\prime}$ with a relative permittivity perturbation $F \delta\left(z-z^{\prime}\right)$.

Integrating the above equation, and using the notation $E_{x}=E$,

$$
\begin{gathered}
\int_{z^{\prime}-0}^{z^{\prime}+0} \frac{\partial^{2} E}{\partial z^{2}} d z=-k^{2} \varepsilon \int_{z^{\prime}-0}^{z^{\prime}+0} E(z) d z-k^{2} F \int_{z^{\prime}-0}^{z^{\prime}+0} \delta\left(z-z^{\prime}\right) E(z) d z, \\
\left.\frac{\partial E}{\partial z}\right|_{z=z^{\prime}+0}-\left.\frac{\partial E}{\partial z}\right|_{z=z^{\prime}-0}=-k^{2} \varepsilon \int_{z^{\prime}-0}^{z^{\prime}+0} E(z) d z-k^{2} F E\left(z^{\prime}\right),
\end{gathered}
$$

where $z^{\prime}+0$ and $z^{\prime}-0$ refer to distances just greater and less than $z^{\prime}$. The first term on the right hand side tends to zero. For the larger $z$ side of $z^{\prime}$ (i.e., the + side), $E\left(z^{\prime}\right)=E_{2} \approx E_{0}$ for the case of a small reflection coefficient $r^{\prime} \ll 1$ from the strain 
pulse (valid if $F \ll 1 / k$, as shown below). Since $r^{\prime} \ll 1$, we do not need to take into account the multiple optical reflections between the strain pulse and the sample surface. For the smaller $z$ side of $z^{\prime}$ (i.e., the - side), $E\left(z^{\prime}\right)=E_{0}+E_{1} \approx E_{0}$. Therefore

$$
\left.\left(\frac{d E_{t}}{d z}-\frac{d\left(E_{i}+E_{r}\right)}{d z}\right)\right|_{z=z^{\prime}}=-F k^{2} E_{0}
$$

where the quantities $E_{i}, E_{r}$ and $E_{t}$ are defined in Fig. 5. This simplifies to

$$
i k_{1} E_{2}-i k_{1} E_{0}+i k_{1} E_{1}=-k^{2} F E_{0} \text {. }
$$

Let $E_{1} / E_{0}=r^{\prime}$ and $E_{2} / E_{0}=t^{\prime}$. Therefore $1-r^{\prime}-t^{\prime}=-i F k^{2} / k_{1}$. Also, $E$ is continuous at $z=z^{\prime}$, so $E_{1}+E_{0}=E_{2}$ at $z=z^{\prime}$. This leads to the relations

$$
1+r^{\prime}=t^{\prime}, \text { and } r^{\prime}=i F k^{2} /\left(2 k_{1}\right) \text {. }
$$

This is the amplitude reflection coefficient for the electric field from the perturbation $\Delta \varepsilon=F \delta\left(z-z^{\prime}\right)$. If the optical absorption in the material is small (i.e. $\left.\kappa \ll n\right)$, so that $k_{1} \approx n k$, this expression for $r^{\prime}$ simplifies to $r^{\prime}=i F \pi /(n \lambda)$. Clearly short wavelengths are reflected better from the discontinuity than long wavelengths. The total reflected electric field from the surface of the material containing the perturbation $\Delta \varepsilon$ is therefore given, in the case of general absorption, by

$$
r E^{(0)}=\left(r_{0}+\frac{i F k^{2} t_{0} \widetilde{t_{0}}}{2 k_{1}} e^{2 i k_{1} z^{\prime}}\right) E^{(0)},
$$

where the Fresnel equations determine the reflection and transmission coefficients:

$$
r_{0}=\frac{k-k_{1}}{k+k_{1}}=\frac{1-n-i \kappa}{1+n+i \kappa} \quad, t_{0}=\frac{2 k}{k+k_{1}}=\frac{2}{1+n+i} \quad, \quad \tilde{t_{0}}=\frac{2 k_{1}}{k+k_{1}}=\frac{2(n+i \kappa)}{1+n+i}
$$

Knowing the response to a $\delta$-function distribution in permittivity allows us to calculate the reflection coefficient, for a general distribution $\Delta \varepsilon(z)$, from an integral of such contributions:

$$
r=r_{0}+\frac{i k^{2}}{2 k_{1}} t_{0} \widetilde{t_{0}} \int_{0}^{\infty} \Delta \varepsilon\left(z^{\prime}\right) e^{2 i k_{1} z^{\prime}} d z^{\prime}=r_{0}+\delta r
$$

where $\delta r$ is the change in $r$. In an isotropic solid we can relate $\Delta \varepsilon$ to the changes in the refractive index induced by the propagating strain as follows:

$$
\varepsilon=(n+i \kappa)^{2} \Rightarrow \Delta \varepsilon(z, t)=\Delta(\tilde{n})^{2} \cong 2 \tilde{n} \Delta \tilde{n}=2(n+i \kappa)\left(\frac{d n}{d \eta}+i \frac{d \kappa}{d \eta}\right) \eta(z, t),
$$

where have abbreviated $\eta_{z z}=\eta$, and the quantities $d n / d \eta$ and $d \kappa / d \eta$ are photoelastic constants (that are in general wavelength dependent)[8,38]. At this point we have introduced a time dependence into the permittivity distribution (assumed here to vary slowly on the time scale of an optical period, a reasonable assumption since $v_{l} \ll c / n$, where $c / n$ is the velocity of light in the solid). The equation for $r$ can be expressed as a relative change in reflectance $\delta r$, where $\delta r=r-r_{0}$ : 


$$
\begin{aligned}
& \frac{\delta r(t)}{r_{0}}=\frac{2 i k}{1-\varepsilon} \int_{0}^{\infty} \Delta \varepsilon\left(z^{\prime}, t\right) e^{2 i k_{1} z^{\prime}} d z^{\prime}=\frac{4 i k \tilde{n}}{1-\tilde{n}^{2}} \int_{0}^{\infty} \Delta \tilde{n}\left(z^{\prime}, t\right) e^{2 i k \tilde{n} z^{\prime}} d z^{\prime} \\
& =\frac{4 i k \tilde{n}}{1-\tilde{n}^{2}}\left(\frac{d n}{d \eta}+i \frac{d \kappa}{d \eta}\right) \int_{0}^{\infty} \eta\left(z^{\prime}, t\right) e^{2 i k \tilde{n} z^{\prime}} d z^{\prime},
\end{aligned}
$$

The reflectivity change of the solid, a real quantity, is given by

$$
\delta R=R-R_{0}=\left|r_{0}+\delta r\right|^{2}-\left|r_{0}\right|^{2} \cong 2 \operatorname{Re}\left(r_{0}{ }^{*} \delta r\right),
$$

where the unperturbed reflectivity is denoted by $R_{0}=r_{0} r_{0}{ }^{*}=\left|r_{0}\right|^{2}$ (* being the complex conjugate). This approximate relation holds provided that $|\delta r|^{2} \ll\left|r_{0}\right|^{2}$. This is generally a good approximation in picosecond laser ultrasonics. In case of measurements on the chromium and nickel films probed at the wavelength $830 \mathrm{~nm}$ far from interband transitions [9], for example, the photoelastic constants $d n / d \eta$ and $d \kappa / d \eta$ as well as the optical constants $n$ and $\kappa$ are less than or around $\sim 5$, and the amplitude of the strain is less than $10^{-4}$. Thus $\left|\delta r / r_{0}\right|$ is on the order of $10^{-5}$ in this case.

Incidentally, we can relate the relative change in reflectivity $\delta R / R_{0}$ to $\delta r / r_{0}$ as follows:

$$
\frac{\delta R}{R_{0}}=2 \operatorname{Re}\left(\frac{r_{0}{ }^{*} \delta r}{r_{0} r_{0}{ }^{*}}\right)=2 \operatorname{Re}\left(\frac{\delta r}{r_{0}}\right)
$$

Equation (9) can be simplified to the form

$$
\delta R(t)=\int_{0}^{\infty} f(z) \eta(z, t) d z,
$$

where

$$
f(z)=f_{0}\left[\frac{d n}{d \eta} \sin \left(\frac{4 \pi n z}{\lambda}-\psi\right)+\frac{d \kappa}{d \eta} \cos \left(\frac{4 \pi n z}{\lambda}-\psi\right)\right] e^{-\frac{z}{\zeta}}
$$

Here, $f_{0}=8 \frac{\omega\left[n^{2}\left(n^{2}+\kappa^{2}-1\right)+\kappa^{2}\left(n^{2}+\kappa^{2}+1\right)\right]^{1 / 2}}{c\left[(n+1)^{2}+\kappa^{2}\right]}$ and $\tan \psi=\frac{\kappa\left(n^{2}+\kappa^{2}+1\right)}{n\left(n^{2}+\kappa^{2}-1\right)}$.

Equation (11) for the reflectance agrees with that originally derived by Thomsen et al.[1]. Equation (9) is a compact form that retains the phase information.[8] The form of $f(z)$, known as the sensitivity function, is an exponentially decaying sinusoid in space. This leads on integration to a temporally localized echo in $\delta R(t)$.

\section{Temporal form of the echoes}

To understand what sort of echo shapes arise, consider a strain pulse returning to a free surface. The strain must have the following form:

$$
\eta(z, t)=\eta_{0}\left(t+\frac{z}{v_{l}}\right)-\eta_{0}\left(t-\frac{z}{v_{l}}\right)
$$

where $\eta_{0}(t)$ is the temporal form of the incident strain pulse when far away from the surface. The minus sign in the second term arises because of the strain inversion on reflection at the free surface (see Fig. 2). At time $t=0$ the strain profile in the solid is 
given by $\eta(z, 0)=\eta_{0}\left(\frac{z}{v_{l}}\right)-\eta_{0}\left(\frac{z}{v_{l}}\right)$. The time $t=0$ corresponds to the moment the strain pulse arrives at the sample surface (at $z=0$ ). At this moment the leading part of the strain pulse has been reflected from the surface and has suffered a sign change. The trailing part of the strain pulse has yet to be reflected from the surface at this time.

Now consider the example of a normalized version of the bipolar strain pulse we considered previously, generated thermoelastically. Let us characterize the temporal dependence of the strain pulse as that corresponding to a time well before it reaches the vacuum-solid interface:

$$
\eta_{0}(s)=\operatorname{sgn}(s) e^{-\frac{v_{l}|s|}{\zeta_{0}}}
$$

Figure 6 shows this strain pulse at times well before $\left(t \ll-\frac{\zeta_{0}}{v_{l}}\right)$ and well after $(t \gg$ $\left.-\frac{\zeta_{0}}{v_{l}}\right)$ its reflection at the free surface. By use of Eqs. (9) and (10) we can calculate the resulting relative reflectivity change. The result is

$$
\frac{\delta R(t)}{R_{0}}=\operatorname{Re}\left[\frac{8 i \tilde{n}}{\left(1-\tilde{n}^{2}\right)\left(\tilde{n}^{2}+\kappa^{\prime 2}\right)} \frac{d \tilde{n}}{d \eta}\left(\kappa^{\prime} e^{\frac{-v_{l}|t|}{\zeta_{0}}}+i \tilde{n} e^{2 i k n v_{l}|t|} e^{\frac{-v_{l}|t|}{\zeta}}\right)\right]
$$

where $\kappa^{\prime}=\kappa_{0} \lambda / \lambda_{0}$, and the refractive index for the pump light of wavelength $\lambda_{0}$ is given by $n_{0}+i \kappa_{0}$.

The reflectivity change consists of the sum of two terms originating from the terms in the round brackets in Eq. (13). The first term, which we call the transient term, has in fact the same time dependence as the time-domain integral of $\eta_{0}(t)$. This will be discussed later on. The second term, which we call the oscillating term, is a damped oscillation with frequency $f_{n}=2 n v_{l} / \lambda$ and decay time $\tau_{n}=\zeta / v_{l}$, quantities that are independent of the form of the strain pulse. The temporal variation is symmetric about $t=0$, a consequence of the antisymmetric form chosen for $\eta_{0}(t)$. An example of a typical reflectivity change is shown in Fig. 7, in which the two terms are also shown separately. The ratio of the transient and oscillating terms depends on the optical constants. Sometimes the oscillations are very heavily damped, as for example in the case of chromium when probing in the near infrared region.[9] 


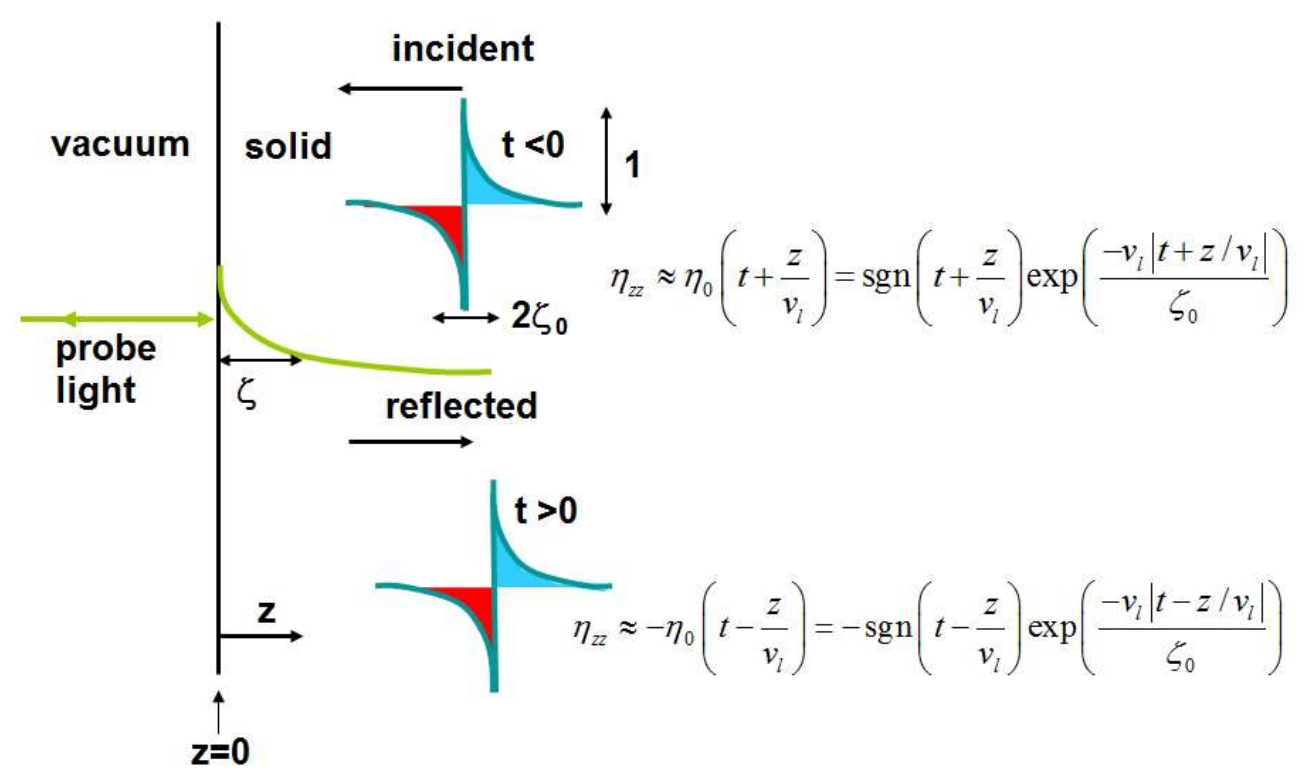

Fig. 6: Plot of a bipolar strain pulse before and after reflection from the free surface of an isotropic solid. The red regions represent compressive strain and the blue regions represent tensile strain. The strain is inverted on reflection. The penetration of the probe light is also shown.

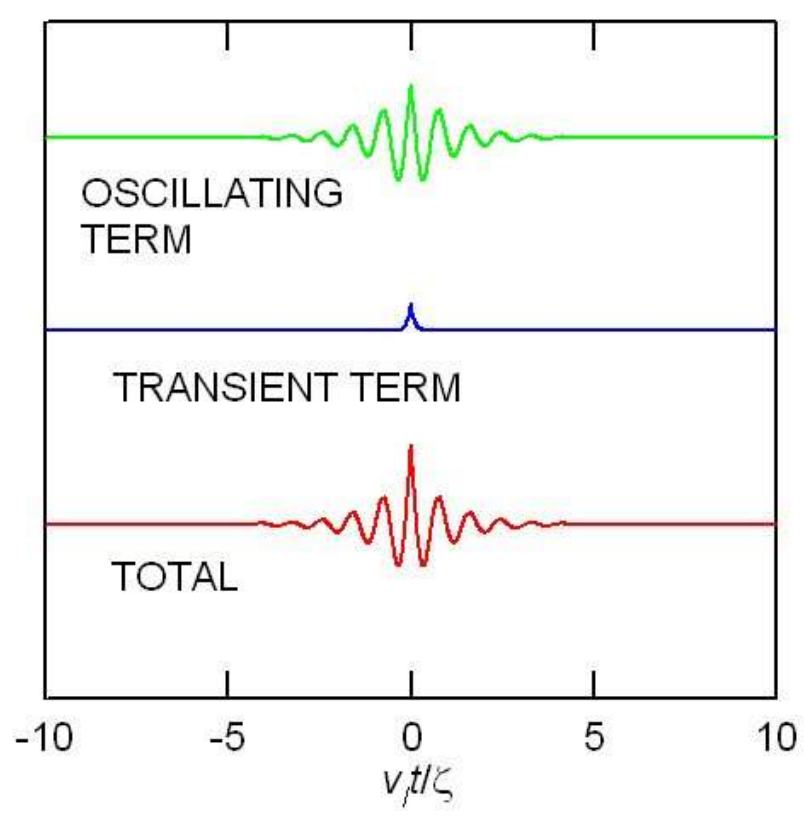

Fig. 7: A typical echo shape plotted as the relative change in reflectivity as a function of normalized time $v_{l} t / \zeta$ for the bipolar strain pulse of Eq. (12) being reflected from the free surface of an isotropic solid. The total contribution is separated into an oscillating term and a transient term. In this simulation $n+i \kappa=$ $1.5+0.2 i, n_{0}+i \kappa_{0}=2+i, \lambda=830 \mathrm{~nm}, \lambda_{0}=415 \mathrm{~nm}, d n / d \eta+i d \kappa / d \eta=$ $1+i$. The spatial decay constant $\zeta_{0}$ of the strain pulse is $\kappa_{0} / \kappa=5$ times smaller than the penetration depth $\zeta$ of the probe light. 
The origin of the oscillating term can be understood by reference to Fig. 8 . The light reflected from the moving strain pulse interferes with the light reflected from the surface of the solid, and this interference varies with time, producing beats. The period of oscillation is determined by the time corresponding to a $2 \pi$ change in the phase difference $\Delta \psi=2 \mathrm{knz}+\theta$ between these two light beams, where $\theta$ is a constant phase. The condition $2 k n z=2 \pi$ yields $f_{n}=2 n v_{l} / \lambda$ when we set $z=v_{l} t$. In fact this frequency is the same frequency that occurs in experiments involving the Brillouin scattering of light (from thermally excited phonons) at normal optical incidence for illumination and detection.[39] For example, when probing chromium with light at wavelength $830 \mathrm{~nm}$ for which $n=3.3, f_{n}=54 \mathrm{GHz}$.[9]

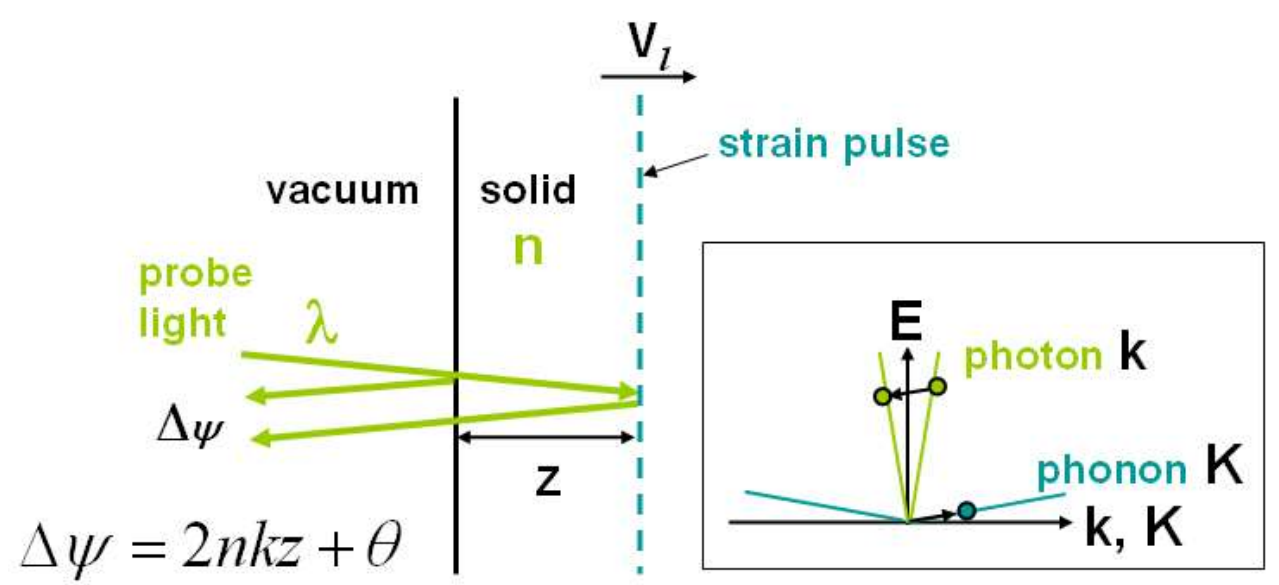

Fig. 8: Schematic diagram showing the origin of the oscillating component for time $t>0$. Because the strain pulse is moving, the interference between the light reflected from the surface and the light reflected from the strain pulse varies with time, leading to beats. The phase difference between the probe light reflected from the surface and from the strain pulse is $\Delta \psi=2 k n z+\theta$, where $\theta$ is a constant phase that depends on the amplitude reflection coefficients of the probe light from the surface and from the strain pulse. The inset shows a schematic diagram of the photon and phonon dispersion relations with the transitions marked (that conserve energy $E$ and wavenumber $k$ ) for the case of a retreating strain pulse.

We can interpret the process on the quantum level as a photon being scattered by a phonon,[40] as shown in Fig. 8 for the case of a strain pulse moving away from the light beam. Applying energy and momentum (k-vector) conservation to the photon [wave number $k^{(i)}$ ] scattering process in which a phonon (wave number $K$ ) is created, we obtain, respectively,

$$
\begin{aligned}
& c_{n}\left|k^{(1)}\right|=c_{n}\left|k^{(2)}\right|+v_{l}|K|, \\
& k^{(1)}=k^{(2)}+K
\end{aligned}
$$

where $c_{n}=c / n$ is the velocity of light in the solid, $c$ is the velocity of light in vacuum, and we have assumed a linear dispersion relation for both the phonons and photons (angular frequency $c_{n} k^{(i)}$ for photons and $v_{l} K$ for phonons). These are reasonable assumptions in general for the experimental conditions in picosecond laser ultrasonics. 
These conservation relations can be satisfied when $k^{(2)}$ is oppositely directly to $k^{(1)}$. Simple algebra leads to

$$
\begin{aligned}
& k^{(2)}=-\frac{c_{n}-v_{l}}{c_{n}+v_{l}} k^{(1)} \\
& K=2 k^{(1)} \frac{c_{n}}{c_{n}+v_{l}} \cong 2 k^{(1)}
\end{aligned}
$$

Equation (14) tells us that the optical angular frequency is slightly downshifted to $\omega=c_{n}\left|k^{(1)}\right|\left(c_{n}-v_{l}\right) /\left(c_{n}+v_{l}\right)$ on reflection from the strain pulse propagating away from the surface. This is none other than the Doppler shift of light reflected from a moving mirror, or Stokes Brillouin scattering. When this light is combined with the light reflected from the sample surface, and the reflectivity $R$ is calculated, the result is the modulation of $R$ at an angular beat frequency $\omega_{n}=2 \pi f_{n}=2 k v_{l} c /\left(c_{n}+v_{l}\right) \cong$ $2 k n v_{l}$, equal to the difference of the two frequencies $\omega=c k\left(c_{n}-v_{l}\right) /\left(c_{n}+v_{l}\right)$ and $c k$. (We obtain a measure of $\delta R(t)$ at a photodetector in practice.) This angular beat frequency of course corresponds to the frequency $f_{n}$ that we derived above through a consideration of the phase difference $\Delta \psi$. Equation (8) also predicts this Doppler shift when we substitute $z^{\prime}=v_{l} t$. For the case of a strain pulse moving towards the incident light beam, similar equations to the above apply except that a phonon propagating towards the surface is annihilated and the optical frequency is upshifted. This corresponds to anti-Stokes Brillouin scattering. The condition of Eq. (15) can also be expressed as $\lambda / n \cong 2 \Lambda$, where $\Lambda$ is the acoustic wavelength and $\lambda / n$ is the optical wavelength in the solid. This condition corresponds to that for Bragg scattering (from an acoustic grating of period $\Lambda$ ).

The term $e^{2 i k_{1} z^{\prime}}=e^{2 i k \tilde{n} z^{\prime}}=e^{2 i k n z^{\prime}} e^{-z^{\prime} / \zeta}$ in Eq. (9) also makes it clear that the extra damping $e^{-z^{\prime} / \zeta}=e^{-v_{l} t / \zeta}$ of the optical beam while inside the solid is responsible for the finite duration $\tau_{n}=\zeta / v_{l}$ of the oscillating component of the echo $\delta R(t)$. (In scattering theory language this damping broadens the photon wave vector distribution of the incident light, allowing the above conservation relation $\Delta k=-K$ to be satisfied for phonon frequencies other than $2 n k$.) This serves to broaden the frequency spectrum of this oscillating component of $\delta R(t)$. For large $\zeta$, that is, for a transparent material, the frequency spectrum of this component becomes very narrow, and many oscillations are seen.[26,41] This will be discussed in more detail below.

An important parameter that determines the echo shape is the phase of the oscillating component of $\delta R(t)$ at $t=0$. This is determined by the form of the strain pulse and by the optical constants of the material. In the present case there is a discontinuity in the gradient of $\delta R(t)$ at $t=0$ owing to the discontinuity in the strain pulse in the form of Eq. (12).

Before continuing it is useful to quickly review the assumptions used in the theory of optical detection so far:

1. The solid is optically and acoustically isotropic.

2. The optical and acoustic beams are considered as infinitely wide plane waves travelling in the $z$ direction.

3. The perturbation of the refractive index is very small.

4. Acoustic and optical dispersion and acoustic losses are neglected.

5. The acoustic frequency is much smaller than the optical frequency (a result of $v_{l} \ll$ c). 
6. The optical and acoustic propagation are governed by linear equations.

7. The optical probe light does not significantly perturb the strain pulse (e.g. by significantly changing its frequency spectrum through the scattering process).

8. The finite optical bandwidth of the probe light pulse is small enough so that the optical constants in Eq. (9) can be assumed to take on unique values.

9. The optical pulse duration is long enough for the different components of the reflected light to interfere coherently.

The relaxation of any of these assumptions leads, of course, to more interesting physics.

\section{Frequency spectrum of the echoes}

By taking the temporal Fourier transform (FT) of $\delta R(t)$ for an echo we may determine its frequency spectrum. Quickly stated, the FT of the transient component of the echo is a measure of the frequency spectrum in the strain pulse (or, more precisely, its integral), whereas the FT of the oscillating component is heavily influenced by the dominant frequency $f_{n}=2 n v_{l} / \lambda$ and the decay time $\tau_{n}=\zeta / v_{l}$ (that determines the spectrum width). Figure 9 (a) shows an example of the modulus of the frequency spectra of the calculated total echo $[\propto \delta R(t)]$ in Fig. 7.

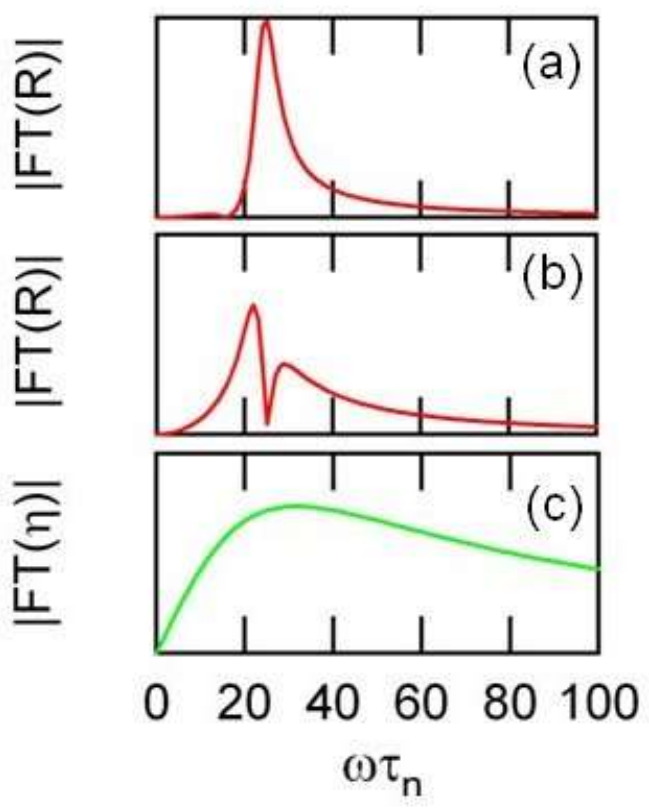

Fig. 9: (a) Plot of the calculated normalized modulus of the frequency spectrum for the reflectivity change $\delta R(t)$ for the parameters stated in the caption of Fig. 7. (b) The same but with $d n / d \eta+i d \kappa / d \eta=1-i$ instead of $1+i$. The scales in (b) and (a) are the same. (c) The normalized modulus of the frequency spectrum for the strain.

Compared to an equivalent spectrum of the strain shown in Fig. 9 (c), the echo spectrum is much narrower. This narrowing in fact increases as the probe beam optical absorption depth $\zeta$ increases. The exact form of the spectrum is very sensitive to the values of the optical constants chosen. In Fig. 9 (b) the spectrum is shown when the 
photoelastic constants are chosen as $d n / d \eta+i d \kappa / d \eta=1-i$ instead of $1+i$. This has the effect of producing a dip in the spectrum as well as extending the spectrum to lower frequencies. When making measurements with different solids one should therefore take care to check what effect the optical detection process has on the observed spectrum of $\delta R(t)$.

\section{Optical phase changes}

Detection in picosecond laser ultrasonics is not limited to reflectivity changes. Equation (9) has both real and imaginary parts. Let us consider the physical significance of the imaginary part. To do this we write $r_{0}=r_{1} \exp \left(i \phi_{1}\right)$, where $r_{0}$ is the probe amplitude reflectance in the absence of the pump pulses, $r_{1}=\left|r_{0}\right|$, and $\phi_{1}=\arg \left(r_{0}\right)$. It is convenient to define $r=r_{1}(1+\rho) \exp \left[i\left(\phi_{1}+\delta \phi\right)\right]$, where $\rho(\ll 1)$ is the relative change in the real part of the reflectance and $\delta \phi(\ll 1)$ is the change in optical phase. A Taylor expansion gives

$$
r=r_{1} e^{i \phi_{1}}(1+\rho+i \delta \phi+\cdots) \cong r_{0}+r_{0}(\rho+i \delta \phi) .
$$

Therefore

$$
\frac{\delta r}{r_{0}} \cong \rho+i \delta \phi
$$

In fact both quantities $\rho(t)$ and $\delta \phi(t)$ are of physical interest and can be measured. A good way to measure them in picosecond laser ultrasonics is to use optical interferometry,[42-44] although the first measurements that were sensitive to $\delta \phi$ were done using probe beam deflection.[2] These measurements showed that a second detection mechanism, independent of the photoelastic effect, was at work: when the strain pulse is reflected from a free surface, the surface moves, producing a change in the phase of the optical probe beam. This motion of the surface results in a bump in the surface, as illustrated in stage 4 of Fig. 1. The induced optical phase change $\delta \phi_{1}$ associated with this surface motion is given, for the case of normal optical incidence from a vacuum, by

$$
\delta \phi_{1}=\frac{4 \pi u(0, t)}{\lambda}=2 k u(0, t)
$$

where $u(0, t)=u_{z}(0, t)$ is the surface displacement (at $z=0$ ) in the $+z$ direction (i.e. into the solid). We must therefore revise Eq. (9) and write

$$
\frac{\delta r(t)}{r_{0}}=\frac{4 i k \tilde{n}}{1-\tilde{n}^{2}} \frac{d \tilde{n}}{d \eta} \int_{0}^{\infty} \eta\left(z^{\prime}, t\right) e^{2 i k \tilde{n} z^{\prime}} d z^{\prime}+2 i k u(t),
$$

where we have defined $u(0, t)=u(t)$ for brevity. The extra term $2 i k u(t)$ in Eq. (16) is purely imaginary, and so does not affect Eq. (11) for $\delta R(t)$. To understand the time dependence of $u(t)$, consider again a general strain pulse in the form

$$
\eta(z, t)=\eta_{0}\left(t+\frac{z}{v_{l}}\right)-\eta_{0}\left(t-\frac{z}{v_{l}}\right) .
$$

From the definition of the surface displacement $u$ as 


$$
u(t)=-\int_{0}^{\infty} \eta\left(z^{\prime}, t\right) d z^{\prime}
$$

one may substitute Eq. (17) into equation (18):

$$
u^{\prime}(t)=v_{l} \int_{-\infty}^{t} \eta_{0}\left(t^{\prime}\right) d t^{\prime}-v_{l} \int_{t}^{\infty} \eta_{0}\left(t^{\prime}\right) d t^{\prime}
$$

Making use of the identity $\frac{d}{d t} \int_{a(t)}^{b(t)} f(x) d x=b^{\prime}(t) f(b(t))-a^{\prime}(t) f(a(t))$, we obtain

$$
\frac{d u(t)}{d t}=-2 v_{l} \eta_{0}(t)
$$

Equation (19) shows that $u(t)$ is proportional to the temporal integral of the strain pulse shape when far from the surface. Alternatively, $d\left(\delta \phi_{1}\right) / d t$ is proportional to $\eta_{0}(t)$.

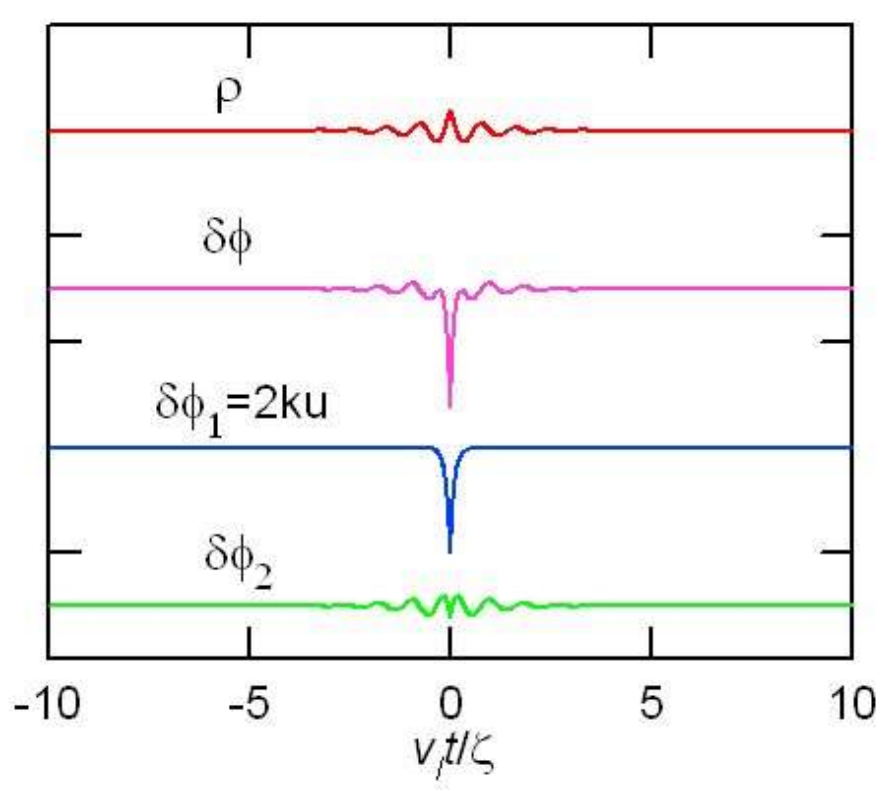

Fig. 10: Typical echo shapes for $\rho$ and $\delta \phi$ plotted as a function of normalized time $v_{l} t / \zeta$ for the bipolar strain pulse of Eq. (12) being reflected from the free surface of an isotropic solid. The separate contributions to $\delta \phi$ are also shown: $\delta \phi_{1}$ from the surface displacement and $\delta \phi_{2}$ from the photoelastic effect. In this simulation $n+$ $i \kappa=1.5+0.2 i, n_{0}+i \kappa_{0}=2+i, \lambda=830 \mathrm{~nm}, \lambda_{0}=415 \mathrm{~nm}$, and $d n / d \eta+$ $i d \kappa / d \eta=1+i$. The spatial decay constant $\zeta_{0}$ of the strain pulse is $\kappa_{0} / \kappa=5$ times smaller than the penetration depth $\zeta$ of the probe light.

Consider now the shape of echoes in picosecond laser ultrasonics when both quantities $\rho(t)$ and $\delta \phi(t)$ are measured. This is routinely done in experiments on metals, semiconductors and dielectrics. $[8,9,15,16]$ The quantity $\rho$ has the same time dependence as $\delta R$, as is obvious from its definition that implies $\rho=\delta R /\left(2 R_{0}\right)$. However, the behaviour of $\delta \phi(t)$ is different. 
Let us return to the bipolar strain pulse of Eq. (12). From Eq. (9), the total relative change in amplitude reflectance is given by

$$
\frac{\delta r(t)}{r_{0}}=\rho+i \delta \phi=\frac{8 i \tilde{n}}{\left(1-\tilde{n}^{2}\right)\left(\tilde{n}^{2}+{\kappa^{\prime}}^{2}\right)} \frac{d \tilde{n}}{d \eta}\left(\kappa^{\prime} e^{-\frac{v_{l}|t|}{\zeta_{0}}}+i \tilde{n} e^{2 i k n v_{l}|t|} e^{-\frac{v_{l}|t|}{\zeta}}\right)+2 i k u(t) .
$$

Figure 10 shows the corresponding variations $\rho(t)$ and $\delta \phi(t)$ for the same optical constants as used in Fig. 7. The phase change is made up of two contributions: $\delta \phi_{1}=$ $2 k u$, the surface displacement contribution, and $\delta \phi_{2}$, a photoelastic contribution arising from the imaginary part of the first term in Eq. (16). These two contributions $\delta \phi_{1}$ and $\delta \phi_{2}$, the total phase change $\delta \phi=\delta \phi_{1}+\delta \phi_{2}$, as well as $\rho$ are shown on the same scale in Fig. 10. The time dependence of $\rho$ is the same as that of $\delta R / R_{0}$ (since they differ only by a factor of 2 ). The time dependence of the photoelastic contribution $\delta \phi_{2}$ is analogous to that of $\rho$, in that it contains an oscillating contribution and a transient contribution. These two contributions are shown in Fig. 11. As for the case of $\rho$ or $\delta R / R_{0}$, the transient contribution shows the same temporal variation as the integral of the strain $u(t)$ (apart from a possible difference in sign). The oscillating component of $\rho$ is $\pi / 2$ out of phase with the oscillating component of $\delta \phi$. This is evident from Eq. (20) since these components arise from the real and imaginary parts in this equation.

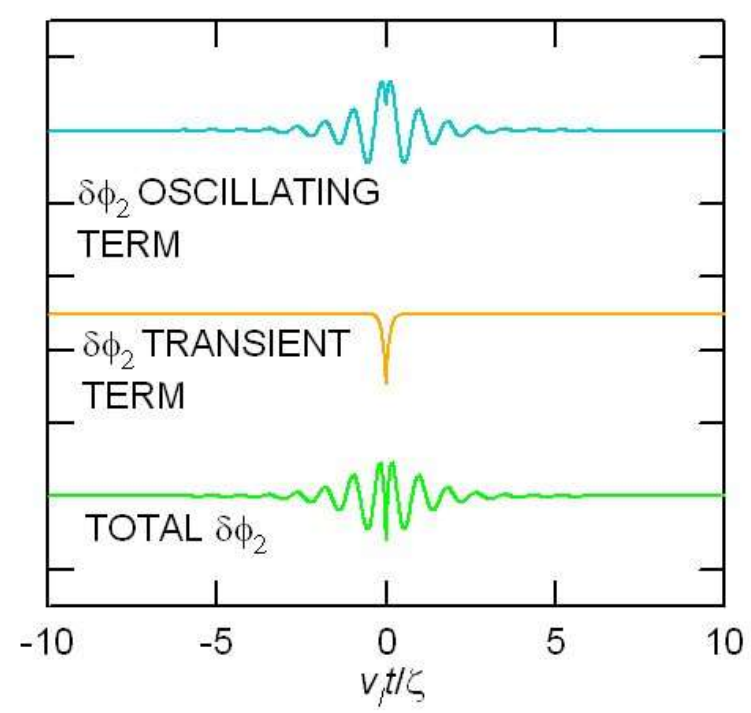

Fig. 11: Echo shape for the photoelastic contribution $\delta \phi_{2}$ to the optical phase change $\delta \phi$ as a function of normalized time $v_{l} t / \zeta$ for the bipolar strain pulse of Eq. (12) being reflected from the free surface of an isotropic solid. The total contribution is separated into an oscillating term and a transient term. In this simulation $n+i \kappa=$ $1.5+0.2 i, \quad n_{0}+i \kappa_{0}=2+i, \lambda=830 \mathrm{~nm}, \quad \lambda_{0}=415 \mathrm{~nm}$, and $d n / d \eta+$ $i d \kappa / d \eta=1+i$. The spatial decay constant $\zeta_{0}$ of the strain pulse is $\kappa_{0} / \kappa=5$ times smaller than the penetration depth $\zeta$ of the probe light.

Equations (16) and (19) suggest that it should be possible to experimentally derive the shape of the strain pulse $\eta_{0}(t)$ from $d(\delta \phi) / d t$ provided that the photoelastic 
contribution to the phase change is very small. This was in fact done using a beam deflection detection method essentially sensitive to the surface displacement variation.[2] A better method for actually separating the photoelastic and surface displacement contributions using oblique probe light incidence was also theoretically and experimentally demonstrated for isotropic solids.[45, 46]

\section{Echo for a $\delta$-function strain pulse}

Much can be gleaned about the physics of echoes by returning to the $\delta$-function strain pulse that we originally considered in Section 2.2 for the detection process: $\eta(z, t)=$ $\delta\left(t+z / v_{l}\right)-\delta\left(t-z / v_{l}\right)$. Unipolar strain pulses similar to this do crop up in practice, both in experiments on thin transparent films on opaque substrates and in experiments on semiconductor quantum wells.[16, 26] From Eqs. (16) and (17) we obtain

$$
\frac{\delta r(t)}{r_{0}}=\frac{4 i k v_{l} \tilde{n}}{\tilde{n}^{2}-1} \frac{d \tilde{n}}{d \eta} \operatorname{sgn}(t) e^{2 i k \tilde{n} v_{l}|t|}+2 i k v_{l} \operatorname{sgn}(t)=D(t)
$$

where $D(t)$ is introduced for brevity. The real and imaginary parts of this function $D(t)$, i.e. $\rho$ and $\delta \phi$, are plotted vs. time in Fig. 12 together with the two contributions to $\delta \phi$ from the surface displacement $\left(\delta \phi_{1}\right)$ and from the photoelastic effect $\left(\delta \phi_{2}\right)$. Because the strain pulse is now symmetric in time, the form of $\rho$ is antisymmetric in time, and likewise for $\delta \phi_{2}$. As in the example of a bipolar strain pulse, there are both oscillating and transient terms in $\rho$ and $\delta \phi$ (although these are not shown separately here). This will be explained in further detail below. As before, the component $\delta \phi_{1}$ mirrors the surface displacement variation $u(t)$. The surface in this case moves inwards as the tensile strain pulse is reflected to a compressive strain pulse, leading to a sudden positive jump in $\delta \phi_{1}$. According to the present theory the surface remains displaced up to $t=+\infty$. [In practice the surface will relax on a time scale $\left(\sim D / v_{R}\right)$ depending on the lateral propagation of surface acoustic waves (of velocity $v_{R}$ ) across the spot diameter (of dimension $D$ )]. 


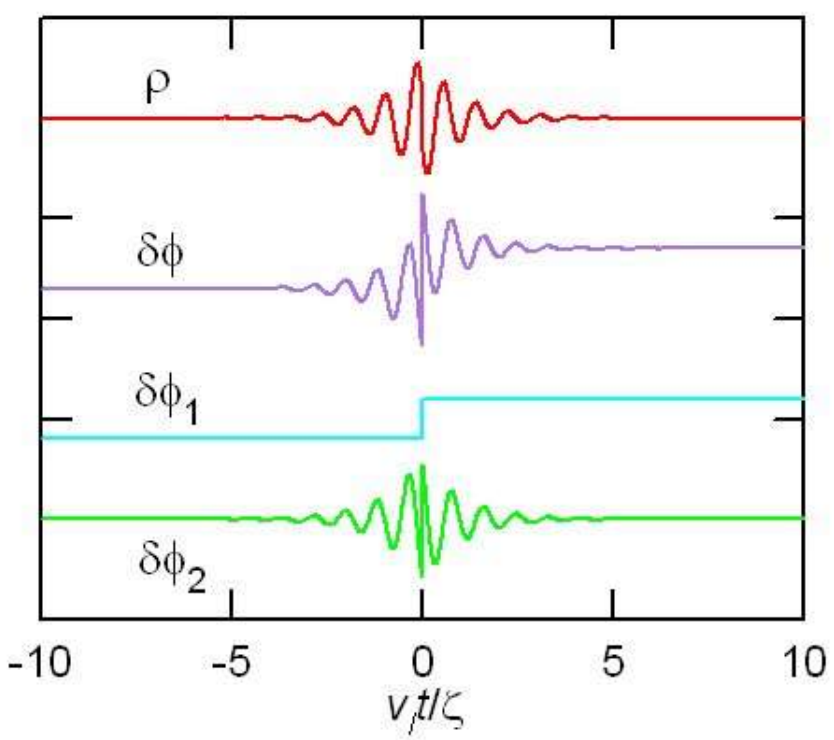

Fig. 12: Typical echo shapes for $\rho$ and $\delta \phi$ plotted as a function of normalized time $v_{l} t / \zeta$ for the $\delta$-function strain pulse being reflected from the free surface of an isotropic solid. The separate contributions to $\delta \phi$ are also shown: $\delta \phi_{1}$ from the surface displacement and $\delta \phi_{2}$ from the photoelastic effect. In this simulation $n+$ $i \kappa=1.5+0.2 i, \lambda=830 \mathrm{~nm}$, and $d n / d \eta+i d \kappa / d \eta=1+i$.

The presence of the transient photoelastic contributions to $\rho$ and $\delta \phi$ for this case can be better understood by considering the time derivative of Eq. (21):

$$
\frac{d}{d t}\left(\frac{\delta r(t)}{r_{0}}\right)=\frac{4 i k^{2} v_{l}^{2} \tilde{n}}{\tilde{n}^{2}-1} \frac{d \tilde{n}}{d \eta} e^{2 i k \tilde{n} v_{l}|t|}+4 i k v_{l} \delta(t)+\frac{\tilde{n}}{\tilde{n}^{2}-1} \frac{d \tilde{n}}{d \eta} \delta(t)=D^{\prime}(t)
$$

The first term in Eq. (22) is the photoelastic oscillation term. The second term arises from the surface displacement. The third term has the same time dependence as the surface displacement term but is photoelastic in origin. This is the transient term.

To understand how this third term is related to the transient contributions to $\rho, \delta \phi$ or $\delta R$ (see Figs. 7 and 11), one should bear in mind that the detection mechanism is linear. Therefore, once the functions $D(t)$ and $D^{\prime}(t)$ are known, the corresponding variations for any shape of strain pulse $\eta_{0}(t)$ can be determined using a convolution operation:

$$
\begin{aligned}
& \frac{\delta r(t)}{r_{0}}=\int_{-\infty}^{\infty} D(\tau) \eta_{0}(t-\tau) d \tau, \\
& \frac{d}{d t}\left(\frac{\delta r(t)}{r_{0}}\right)=\int_{-\infty}^{\infty} D^{\prime}(\tau) \eta_{0}(t-\tau) d \tau .
\end{aligned}
$$

The convolution of the strain pulse $\eta_{0}(t)$ with the $\delta$-function in the third term in Eq. (22) will produce the same function $\eta_{0}(t)$ (apart from a multiplicative constant). On integration we will therefore obtain a transient contribution to $\rho$ and $\delta \phi$ proportional to the temporal integral of the strain.

This analysis can also give us some insight into the physical meaning of the sensitivity function $f(z)$ introduced by Thomsen et al., as defined in Eq. (11). If we introduce our $\delta$-function strain pulse $\eta(z, t)=\delta\left(t+z / v_{l}\right)-\delta\left(t-z / v_{l}\right)$ into this equation, we find 


$$
\delta R(t)=v_{l} f\left(v_{l}|t|\right) \operatorname{sgn}(t) /
$$

This implies that

$$
\operatorname{Re}(D(t))=\frac{v_{l}}{2 R_{0}} f\left(v_{l}|t|\right) \operatorname{sgn}(t) .
$$

Equation (23) tells us that the response $\operatorname{Re}(D(t))$ to a $\delta$-function strain pulse is proportional to two sensitivity functions $f(z)$ placed back to back in an antisymmetric fashion, with $z$ replaced by $v_{l}|t|$. This gives a satisfying physical interpretation of the sensitivity function.

\section{Amplitude-phase plots of echoes}

Since a full measurement of an echo requires both amplitude and phase measurements, one way of viewing an echo would be to plot the amplitude against the phase. Figure 13 (a) shows a plot of $\rho$ vs. $\delta \phi_{2}$ (i.e. with the surface displacement contribution removed). The centre of the spiral corresponds to the points $t= \pm \infty$, whereas the free end of the spiral corresponds to the point $t=0$. The vertical and horizontal scales are the same, owing to the $\pi / 2$ phase difference between the oscillating components of $\rho$ and $\delta \phi_{2}$. (The transient components of $\rho$ and $\delta \phi_{2}$ are relatively small.) The rainbow colour is added to convey a measure of $|t|$.
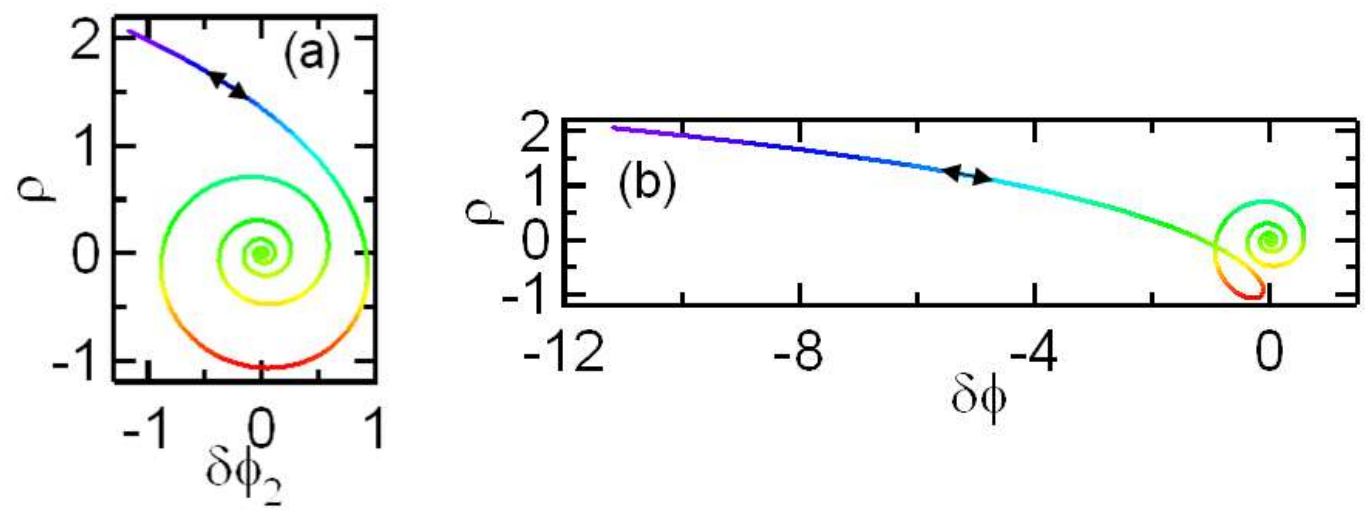

Fig. 13: Echo shapes represented as amplitude-phase plots for a bipolar strain pulse: (a) $\rho$ vs. the photoelastic contribution $\delta \phi_{2}$ to the optical phase change. (b) $\rho$ vs. $\delta \phi$. In this simulation $n+i \kappa=1.5+0.2 i, n_{0}+i \kappa_{0}=2+i, \lambda=830 \mathrm{~nm}, \lambda_{0}=$ $415 \mathrm{~nm}$, and $d n / d \eta+i d \kappa / d \eta=1+i$. The spatial decay constant $\zeta_{0}$ of the strain pulse is $\kappa_{0} / \kappa=5$ times smaller than the penetration depth $\zeta$ of the probe light.

Figure 13(b) shows a plot of $\rho$ vs. $\delta \phi$. The effect of including the displacement term is to displace the end of the spiral. The shape of the curve at large $|t|$ is not altered because the displacement contribution dies out quickly with increasing $|t|$.

Figure 14 shows similar plots for the $\delta$-function pulse. The curves are more complicated because the echo shapes are no longer an even function in time and because of the abrupt changes at $t=0$. 
These plots are possibly the best way to appreciate an echo in picosecond laser ultrasonics at a single glance, although if viewed for too long the psychedelic spirals may jar the nerves.
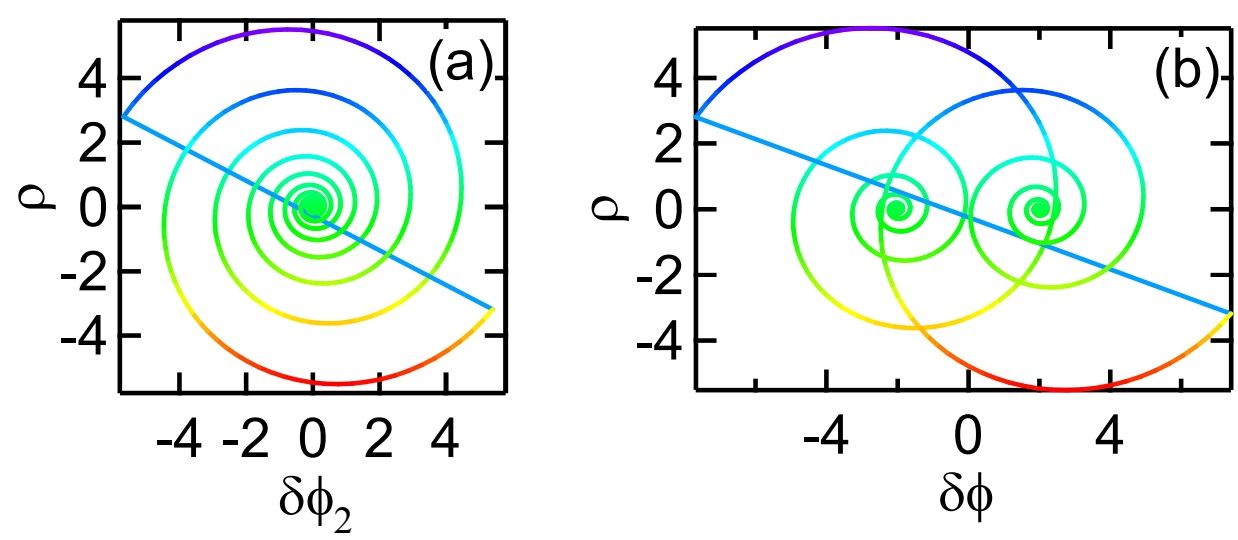

Fig. 14: Echo shapes represented as amplitude-phase plots for a $\delta$-function strain pulse: (a) $\rho$ vs. the photoelastic contribution $\delta \phi_{2}$ to the optical phase change. (b) $\rho$ vs. $\delta \phi$. In this simulation $n+i \kappa=1.5+0.2 i, \lambda=830 \mathrm{~nm}$, and $d n / d \eta+$ $i d \kappa / d \eta=1+i$. 


\section{Optical detection in perturbed multilayers and photonic crystals}

\section{Electromagnetic wave equation}

The reflection of light from multilayer thin film structures is an important problem for a variety of applications. When using such samples in picosecond laser ultrasonics we may need to account for the inhomogeneous modulation of the permittivity over several layers along the stacking direction induced by a propagating strain pulse. Many commercially and physically important samples such as semiconductor heterostructures or multilayer metal stacks are partially transparent at visible or near-visible optical wavelengths when film thicknesses are in the nanometre to micron range.

For one- or two-layer structures, simple techniques have been developed to deal with the problem of multiple optical reflections when calculating the modulation in reflectance or transmittance.[26, 47, 48]. For samples with more layers, a general theoretical approach based on Green's functions has been developed, allowing the calculation of both reflectance and transmittance changes at normal optical incidence.[20] An outline of this approach is given here.

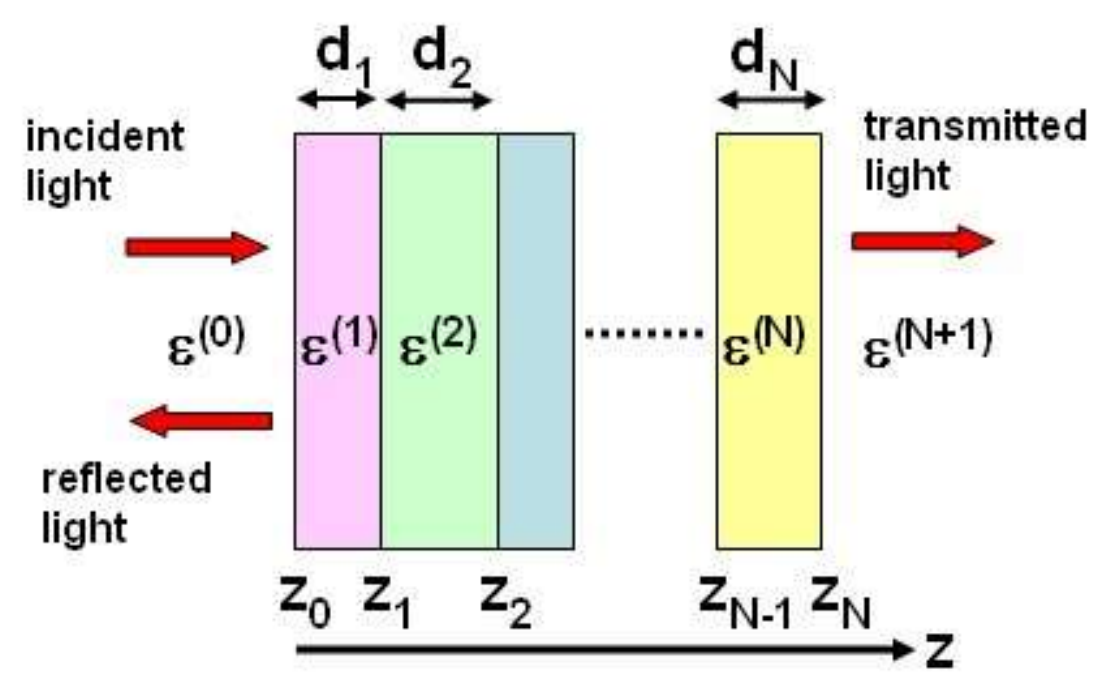

Fig. 15: A general multilayer structure consisting of $N$ layers on a substrate. Each layer is considered to be optically isotropic before perturbation. We define $z_{0}=0$.

Figure 15 shows the sample geometry in the unperturbed state, consisting of $N$ parallel layers on a substrate. The medium in front of the sample is a transparent isotropic material rather than a vacuum. All materials are considered to be optically and acoustically isotropic. We also assume that the total thickness of the sample is much shorter than the spatial length of the incident optical pulse in the $z$ direction (typically $\sim 30 \mu \mathrm{m}$ for a $100 \mathrm{fs}$ optical pulse). This implies that the light always interferes coherently.

The problem is to find the change in reflectance or transmittance of this structure when it contains an arbitrary distribution of longitudinal strain in the stacking $(z)$ direction. There may also be strain in the incident medium or in the substrate, but not at $z= \pm \infty$. With the linear approximation $\mathbf{D}=\varepsilon_{0} \tilde{\varepsilon}(\mathbf{r}) \mathbf{E}$, we start by invoking the Maxwell's equations for $\mathbf{E}$ and $\mathbf{B}$ in an medium with an inhomogeneous quasistatic 
variation in the complex permittivity:

$$
\begin{aligned}
& \varepsilon_{0} \nabla \cdot(\tilde{\varepsilon}(\mathbf{r}) \mathbf{E})=0, \\
& \nabla \cdot \mathbf{B}=0 \\
& \nabla \times \mathbf{E}=-\partial \mathbf{B} / \partial t \\
& \nabla \times \mathbf{B}=\varepsilon_{0} \mu_{0} \tilde{\varepsilon}(\mathbf{r}) \partial \mathbf{E} / \partial t
\end{aligned}
$$

where $\tilde{\varepsilon}(\mathbf{r})$ is the $3 \times 3$ complex relative permittivity tensor which is a function of position r. $\widetilde{\varepsilon}(\mathbf{r})$ is $\omega$ dependent in general. Combining these equations leads to

$$
\nabla \times(\nabla \times \mathbf{E})=\nabla(\nabla \cdot \mathbf{E})-\nabla^{2} \mathbf{E}=-\frac{\partial}{\partial t}(\nabla \times \mathbf{B})=-\varepsilon_{0} \mu_{0} \tilde{\varepsilon}(\mathbf{r}) \frac{\partial^{2} \mathbf{E}}{\partial t^{2}},
$$

or

$$
\left(\nabla^{2}-\operatorname{grad} \operatorname{div}\right) \mathbf{E}(\mathbf{r}, t)=\mu_{0} \frac{\partial^{2}}{\partial t^{2}} \mathbf{D}(\mathbf{r}, t)
$$

Under the assumption of incidence with monochromatic light at frequency $\omega$, we can omit the common term $e^{-i \omega t}$ and simplify using the equation relating $\mathbf{D}$ and $\mathbf{E}$ :

$$
\left(\nabla^{2}-\operatorname{grad} \operatorname{div}+k^{2} \widetilde{\varepsilon}(\mathbf{r})\right) \mathbf{E}(\mathbf{r})=0
$$

where, as usual, $k=\omega \sqrt{\varepsilon_{0} \mu_{0}}$ is the vacuum wave number.

The 1D geometry of this multilayer problem allows further simplification. Writing $\mathbf{E}(\mathbf{r})=\mathbf{E}(z)$ for the case of normal incidence, and remembering that $\tilde{\varepsilon}$ is a $3 \times 3$ tensor that depends in this case only on a single spatial coordinate $(z)$,

$$
\left[\left(\begin{array}{ccc}
\partial^{2} / \partial z^{2} & 0 & 0 \\
0 & \partial^{2} / \partial z^{2} & 0 \\
0 & 0 & 0
\end{array}\right)+k^{2} \widetilde{\varepsilon}(z)\right] \mathbf{E}(z)=0
$$

To go further we must again consider the photoelastic effect.

\section{Perturbation of the permittivity tensor}

The relative permittivity tensor component of interest, $\varepsilon_{x x}$, can be divided into an unperturbed part $\varepsilon_{h}$ (or homogeneous part) and a perturbed part $\varepsilon_{i h}$ (or inhomogeneous part). The unperturbed part is illustrated in Fig. 15. The perturbed part depends partly on the photoelastic effect. Let us take a look at the general equations describing this effect[38,49]:

$$
\Delta \varepsilon_{i j}=P_{i j k l} \eta_{k l},
$$

where $P_{i j k l}$ is a photoelastic constant tensor and $\eta_{k l}$ is the strain tensor. Bear in mind that both refractive index and extinction coefficient can be modified by strain, so $P_{i j k l}$ is a complex quantity. In abbreviated notation $(1=x x, 2=y y, 3=z z, 4=y z=$ $z y, 5=z x=x z, 6=x y=y x), \Delta \varepsilon_{I}=P_{I J} \eta_{J}$. For an isotropic solid, this equation can be written as[38] 


$$
\left(\begin{array}{c}
\Delta \varepsilon_{1} \\
\Delta \varepsilon_{2} \\
\Delta \varepsilon_{3} \\
\Delta \varepsilon_{4} \\
\Delta \varepsilon_{5} \\
\Delta \varepsilon_{6}
\end{array}\right)=\left(\begin{array}{cccccc}
P_{11} & P_{12} & P_{12} & 0 & 0 & 0 \\
P_{12} & P_{11} & P_{12} & 0 & 0 & 0 \\
P_{12} & P_{12} & P_{11} & 0 & 0 & 0 \\
0 & 0 & 0 & P_{44} & 0 & 0 \\
0 & 0 & 0 & 0 & P_{44} & 0 \\
0 & 0 & 0 & 0 & 0 & P_{44}
\end{array}\right)\left(\begin{array}{l}
\eta_{1} \\
\eta_{2} \\
\eta_{3} \\
\eta_{4} \\
\eta_{5} \\
\eta_{6}
\end{array}\right)
$$

where $P_{44}=\left(P_{11}-P_{12}\right) / 2$. In our case of longitudinal strain propagating in the $Z$ direction, $\eta_{3}=\eta_{z z}$ is the only non-zero strain component. So for our geometry of normal incidence and $x$-polarized light, $\Delta \varepsilon_{1}=\Delta \varepsilon_{x x}=P_{12} \eta_{3}=P_{12} \eta_{z z}=P_{12} \eta$. In fact

$$
\tilde{\varepsilon}_{p e}=\eta\left(\begin{array}{ccc}
P_{12} & 0 & 0 \\
0 & P_{12} & 0 \\
0 & 0 & P_{11}
\end{array}\right)
$$

defines the photoelastic perturbation to $\tilde{\varepsilon}$. The longitudinal strain distribution evidently does not induce off-diagonal components in $\tilde{\varepsilon}$, so Eq. (25) reduces to two independent and equivalent wave equations for $x$ - and $y$-polarized light. Without loss of generality we only require a solution for $E_{x}(z)$, depending on $\varepsilon_{x x}$ only. (This corresponds to the incident light being polarized in the $x$ direction.)

We may equivalently express the perturbation in terms of the refractive index $\tilde{n}=$ $\sqrt{\varepsilon_{x x}}$ :

$$
\Delta \tilde{n}=\frac{P_{12}}{2 \tilde{n}} \eta=\left(\frac{d n}{d \eta}+i \frac{d \kappa}{d \eta}\right) \eta=\frac{d \tilde{n}}{d \eta} \eta
$$

The quantity $d \tilde{n} / d \eta$ in this equation is of course the same as the one that arose in Eqs. (9) and (16). The photoelastic effect occurs in each of the $N+2$ regions in the multilayer sample; the contribution to the perturbed part, $\varepsilon_{i h}$, of the $x x$ component of the permittivity tensor is given by

$$
\varepsilon_{p e}=P_{12}^{(n)} \eta(z) \text { in the } n \text {-th layer. }
$$

In addition, we should also consider the motion of the interfaces due to the strain. At any point in the sample the displacement is

$$
u(z)=-\int_{z}^{\infty} \eta\left(z^{\prime}\right) d z^{\prime} .
$$

We can represent the interface motions (at $z=z_{n}$ ) by effective changes in the permittivity in the region of the interfaces. The changes themselves are large, but their regions of application are restricted to tiny slices close to the interfaces, because the interface displacements are in general very small (e.g., $\sim 1 \mathrm{pm})$ compared to the layer thicknesses. Let us denote the associated change in the $x x$ component of the permittivity tensor $\varepsilon_{i h}$ by $\varepsilon_{d}$, where

$$
\varepsilon_{d}=
$$




$$
\left\{\begin{array}{ll}
\varepsilon^{(n)}-\varepsilon^{(n+1)} & \text { for } u\left(z_{n}\right)>0, z_{n}<z<z_{n}+u\left(z_{n}\right), \\
\varepsilon^{(n+1)}-\varepsilon^{(n)} & \text { for } u\left(z_{n}\right)<0, z_{n}+u\left(z_{n}\right)<z<z_{n}
\end{array},\right.
$$

These perturbations are shown schematically in Fig. 16 for the case in which $\varepsilon_{i h}$ is a real quantity. The perturbation $\varepsilon_{d}(z)$ appears as a top hat shape. In the example shown, $u\left(z_{1}\right)$ is positive, leading to $\varepsilon_{d}=\varepsilon^{(1)}-\varepsilon^{(2)}>0$. We must deal with the combined perturbation $\varepsilon_{i h}=\varepsilon_{p e}+\varepsilon_{d}$.

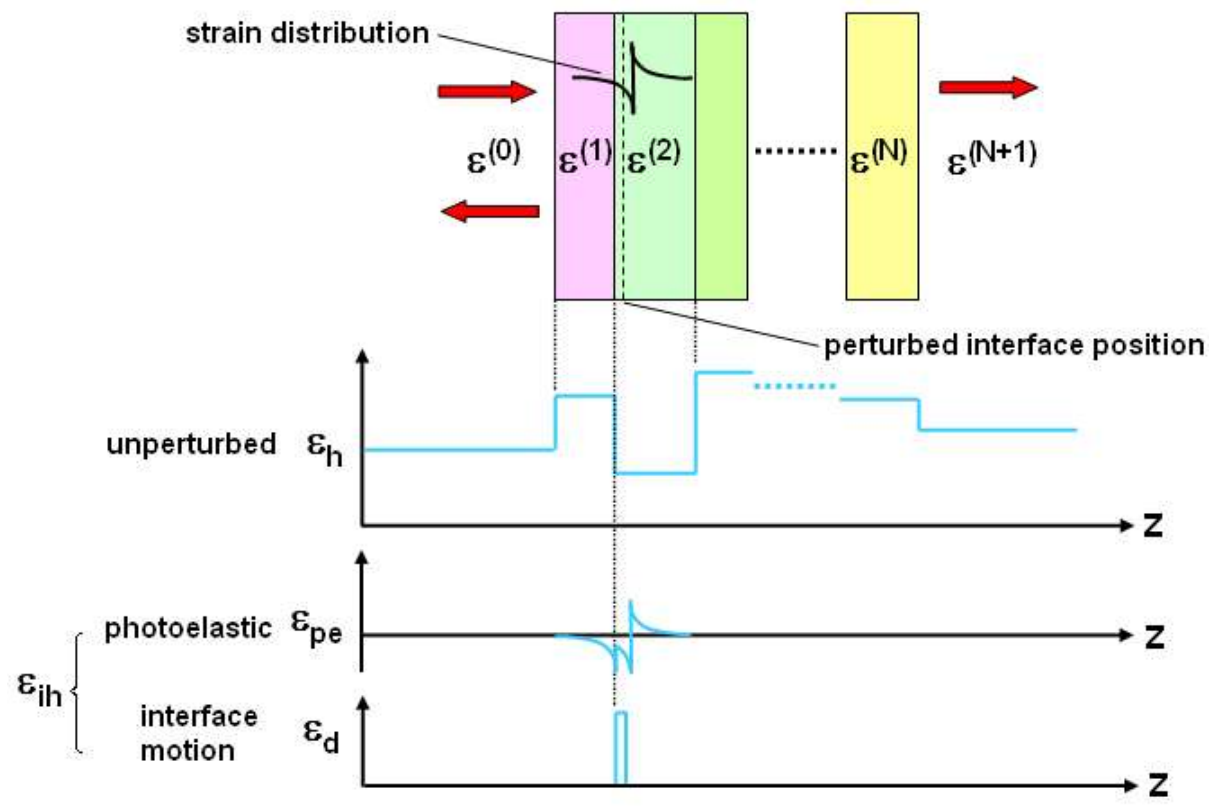

Fig. 16: Schematic diagram of the dielectric constant distributions in the presence of a longitudinal strain pulse in layers 1 and 2 . The vertical dashed line in layer 2 shows the perturbed interface position. The top graph show the unperturbed distribution of the relative permittivity $\varepsilon_{h}$. The middle graph shows the change in permittivity $\varepsilon_{p e}$ caused by the photoelastic effect. The bottom plot shows the change in permittivity $\varepsilon_{d}$ caused by the interface motion. Here $\varepsilon_{i h}=\varepsilon_{p e}+\varepsilon_{d}$. The values here are assumed to be real, and the magnitude of $\varepsilon_{p e}$ is greatly exaggerated.

\section{Solution of the wave equation}

We proceed by solving the wave equation corresponding to Eq. (25) with $\varepsilon_{x x}(z)=$ $\varepsilon_{h}(z)$ :

$$
\left[\frac{\partial^{2}}{\partial z^{2}}+k^{2} \varepsilon_{h}(z)\right] E_{0}(z)=0
$$

where $E_{0}$ is the solution for $E_{x}$ in the absence of strain. This can be done very straightforwardly by considering the boundary conditions at each interface and using a transfer matrix method. $[8,50,51]$ We briefly review this method here. The solution for $E_{0}$ in the $n$th layer has the form

$$
a_{n} e^{i k_{n} Z_{n}}+b_{n} e^{-i k_{n} Z_{n}}
$$


where $Z_{n}=z-z_{n-1}(n \geq 1), Z_{0}=z, z_{-} 0=0$, and $k_{n}=\sqrt{\varepsilon^{(n)}} k$. The electric field amplitudes $a_{n}$ and $b_{n}$ can be determined by the following procedure. The boundary conditions on $\mathbf{E}$ and $\mathbf{H}\left(=\boldsymbol{\nabla} \times \mathbf{E} /\left(i \omega \mu_{0}\right)\right)$ at the first interface $(z=0)$ require continuity of the parallel components of these vectors. These conditions lead to the matrix equation

$$
\left(\begin{array}{cc}
1 & 1 \\
k_{0} & -k_{0}
\end{array}\right)\left(\begin{array}{l}
a_{0} \\
b_{0}
\end{array}\right)=\left(\begin{array}{cc}
1 & 1 \\
k_{1} & -k_{1}
\end{array}\right)\left(\begin{array}{l}
a_{1} \\
b_{1}
\end{array}\right)
$$

or, in compact form, $\mathbf{M}_{0} \mathbf{a}_{0}=\mathbf{M}_{1} \mathbf{a}_{1}$. The Fresnel equations for reflection at normal incidence immediately follow (see Figs. 4 and 5). At $z=z_{n}$ the appropriate relation is $\mathbf{M}_{n} \mathbf{Q}_{n} \mathbf{a}_{n}=\mathbf{M}_{n+1} \mathbf{a}_{n+1}$, where

$$
\mathbf{M}_{n}=\left(\begin{array}{cc}
1 & 1 \\
k_{n} & -k_{n}
\end{array}\right), \mathbf{Q}_{n}=\left(\begin{array}{cc}
e^{i k_{n} d_{n}} & 0 \\
0 & e^{-i k_{n} d_{n}}
\end{array}\right)
$$

The matrix $\mathbf{Q}_{n}$ accounts for the thickness $d_{n}$ of the $n$th layer (where $n \geq 1$ ). In order to calculate the matrix $\mathbf{M}$ that relates the fields incident on the sample to those transmitted to the substrate, that is $\mathbf{a}_{0}=\mathbf{M} \mathbf{a}_{n+1}$, we iterate these equations to obtain

$$
\mathbf{M}=\mathbf{M}_{0}{ }^{-1}\left(\sum_{j=1}^{N} \mathbf{M}_{j} \mathbf{Q}_{j}^{-1} \mathbf{M}_{j}^{-1}\right) \mathbf{M}_{N+1} .
$$

The reflectance of the whole structure is then simply given by $r_{0}=b_{0} / a_{0}=M_{21} / M_{11}$. Furthermore, by stopping the above series at a given layer $n$, we may determine the electric field amplitudes $a_{n}$ and $b_{n}$ for every layer, and hence determine $E_{0}(z)$.

From Eq. (25), the $x$ component of the electric field can be obtained from

$$
\left[\frac{\partial^{2}}{\partial z^{2}}+k^{2}\left(\varepsilon_{h}(z)+\varepsilon_{i h}(z)\right)\right] E(z)=0 .
$$

To do this, we define a Green's function $G\left(z, z^{\prime}\right)$ as follows:

$$
\left[\frac{\partial^{2}}{\partial z^{2}}+k^{2} \varepsilon_{h}(z)\right] G\left(z, z^{\prime}\right)=-\delta\left(z-z^{\prime}\right)
$$

The form for $G$ will be given shortly. First let us express the solution of Eq. (29) in terms of $G$ :

$$
\begin{array}{r}
E(z)=E_{0}(z)+k^{2} \int_{-\infty}^{\infty} G\left(z, z^{\prime}\right) \varepsilon_{i h}\left(z^{\prime}\right) E\left(z^{\prime}\right) d z^{\prime} \\
\cong E_{0}(z)+k^{2} \int_{-\infty}^{\infty} G\left(z, z^{\prime}\right) \varepsilon_{i h}\left(z^{\prime}\right) E_{0}\left(z^{\prime}\right) d z^{\prime},
\end{array}
$$

where the approximation $E_{0}\left(z^{\prime}\right) \cong E\left(z^{\prime}\right)$ in the integral holds for first-order perturbation theory. This relation can be verified by applying the operator $\left(\partial^{2} / \partial z^{2}\right)+$ $k^{2} \varepsilon_{h}(z)$ to both sides of Eq. (31) and making use of Eqs. (28) and (30). (Note that this operator does not act on the terms in the integral depending only on $z^{\prime}$.) Equation (31) implies that $G\left(z, z^{\prime}\right)$ governs the response at $z$ caused by a localized disturbance (i.e. a $\delta$-function change in permittivity) at $z^{\prime}$.

The Green's function can be found by considering its limiting properties at $\mathrm{z}=$ $\mathrm{z}^{\prime}$ and $z= \pm \infty$, and by realising that $G$ obeys the same boundary conditions as an 
electric field at the interfaces. The details are given in Ref. [8]. The result for the region of interest is

$$
G\left(z, z^{\prime}\right)=\frac{i}{2 k_{0} a_{0}} e^{-i k_{0} z} E_{0}\left(z^{\prime}\right) \text { for } z^{\prime}>z, z<0
$$

where $k_{0}=\sqrt{\varepsilon^{(0)}} k$ is the wave number in medium 0 . (It can be shown that the reciprocity relation $G\left(z, z^{\prime}\right)=G\left(z^{\prime}, z\right)$ holds, and this helps in deriving $G$.[8]) The next step is to substitute for $G$ in Eq. (31) at a point $z<0$, including both contributions to $\varepsilon_{i h}$. We obtain

$$
\begin{aligned}
E(z)=a_{0} e^{i k_{0} z}+b_{0} & e^{-i k_{0} z}+\frac{i k^{2} e^{-i k_{0} z}}{2 k_{0} a_{0}}\left\{\int_{z}^{0} P_{12}^{(0)} \eta\left(z^{\prime}\right)\left[a_{0} e^{i k_{0} z^{\prime}}+b_{0} e^{-i k_{0} z^{\prime}}\right]^{2} d z^{\prime}\right. \\
& +\sum_{n=1}^{N+1} \int_{0}^{d_{n}} P_{12}^{(n)} \eta\left(z^{\prime}+z_{n-1}\right)\left[a_{n} e^{i k_{n} z^{\prime}}+b_{n} e^{-i k_{n} z^{\prime}}\right]^{2} d z^{\prime} \\
& \left.+\sum_{n=1}^{N+1}\left(a_{n}+b_{n}\right)^{2}\left[\varepsilon^{(n-1)}-\varepsilon^{(n)}\right] u\left(z_{n-1}\right)\right\} .
\end{aligned}
$$

The relative reflectance change is therefore given by

$$
\begin{aligned}
\frac{\delta r}{r_{0}}=\frac{i k^{2}}{2 k_{0} a_{0} b_{0}}\{ & \left\{\int_{z}^{0} P_{12}^{(0)} \eta\left(z^{\prime}\right)\left[a_{0} e^{i k_{0} z^{\prime}}+b_{0} e^{-i k_{0} z^{\prime}}\right]^{2} d z^{\prime}\right. \\
& +\sum_{n=1}^{N+1} \int_{0}^{d_{n}} P_{12}^{(n)} \eta\left(z^{\prime}+z_{n-1}\right)\left[a_{n} e^{i k_{n} z^{\prime}}+b_{n} e^{-i k_{n} z^{\prime}}\right]^{2} d z^{\prime} \\
& \left.+\sum_{n=1}^{N+1}\left(a_{n}+b_{n}\right)^{2}\left[\varepsilon^{(n-1)}-\varepsilon^{(n)}\right] u\left(z_{n-1}\right)\right\},
\end{aligned}
$$

where $r_{0}=b_{0} / a_{0}$ is the reflectance of the whole structure, and the point $z \quad(<0)$ is to the left of all strain perturbations. The first term in Eq. (32) accounts for the photoelastic effect in the incident medium $(n=0)$, the second term accounts for the photoelastic effect in the multilayer and substrate, and the third term accounts for the interface motion. This relation is equivalent to the formula quoted by Perrin et al.[42]

In the simple case of a sample consisting of an incident medium (0) and a substrate (1), Eq. (32) reduces to Eq. (16). Another way to see this is to substitute a $\delta$-function change in permittivity at a point $z^{\prime \prime}>0$ in Eq. (31), so that $\varepsilon_{i h}=\delta\left(z^{\prime}-z^{\prime \prime}\right)$. The result is

$$
E(z)-E_{0}(z)=k^{2} E_{0}\left(z^{\prime \prime}\right) G\left(z, z^{\prime \prime}\right)=\frac{i k^{2}}{2 k_{0} a_{0}} E_{0}^{2}\left(z^{\prime \prime}\right) e^{-i k_{0} z}
$$

As expected, $G\left(z, z^{\prime \prime}\right)$ governs the response at $z$ to a local change in permittivity at $z^{\prime \prime}$. We also know that $E(z)-E_{0}(z)=e^{-i k_{0} z} \delta b_{0}$, where $\delta b_{0}$ is the change in the reflected electric field amplitude $b_{0}$ due to the perturbation for a point $z$ to the left of all perturbations. By reference to Fig. $9, E_{0}^{2}\left(z^{\prime \prime}\right)=t_{0}^{2} a_{0}^{2} e^{2 i k_{1} z^{\prime \prime}}=$ $t_{0} \tilde{t}_{0} a_{0}{ }^{2} e^{2 i k_{1} z^{\prime \prime}} k_{1} / k_{0}$, where use has been made of the Fresnel equations. Using the relation $r-r_{0}=\delta b_{0} / a_{0}$, and setting $z^{\prime \prime}=z^{\prime}$ as the position for the perturbation, we obtain Eq. (8) when $F=1$, as expected. Incidentally, the relation $E_{0}\left(z^{\prime}\right)=t_{0} a_{0} e^{i k_{1} z^{\prime}}=2 k_{0} a_{0} e^{i k_{1} z^{\prime}} /\left(k_{0}+k_{1}\right)$ shows that for this simple case of two media,

$$
G\left(z, z^{\prime}\right)=\frac{i}{k_{0}+k_{1}} e^{i\left(k_{1} z^{\prime}-k_{0} z\right)} \text { for } z^{\prime}>z, z<0
$$


The same general theoretical method can also be applied to calculate the modulation in transmission through a multilayer.[8]

\section{Light modulation in a transparent-metal photonic crystal}

An interesting example of the application of the above theory is the ultrafast generation of sound in a photonic crystal. We present here some preliminary results on a particular type of 1D photonic crystal in the form of a 'transparent-metal' structure.[52] It consists of a symmetric periodic structure with four unit cells, with each unit cell composed of a silver layer (15 nm thick) sandwiched between two identical dielectric $\mathrm{TiO}_{2}$ layers $(32$ $\mathrm{nm}$ thick), as shown in Fig. 17 (a). (A thin $1 \mathrm{~nm}$ layer of Ti was used at the $\mathrm{Ag}-\mathrm{TiO}_{2}$ interface to prevent oxidation.[53])
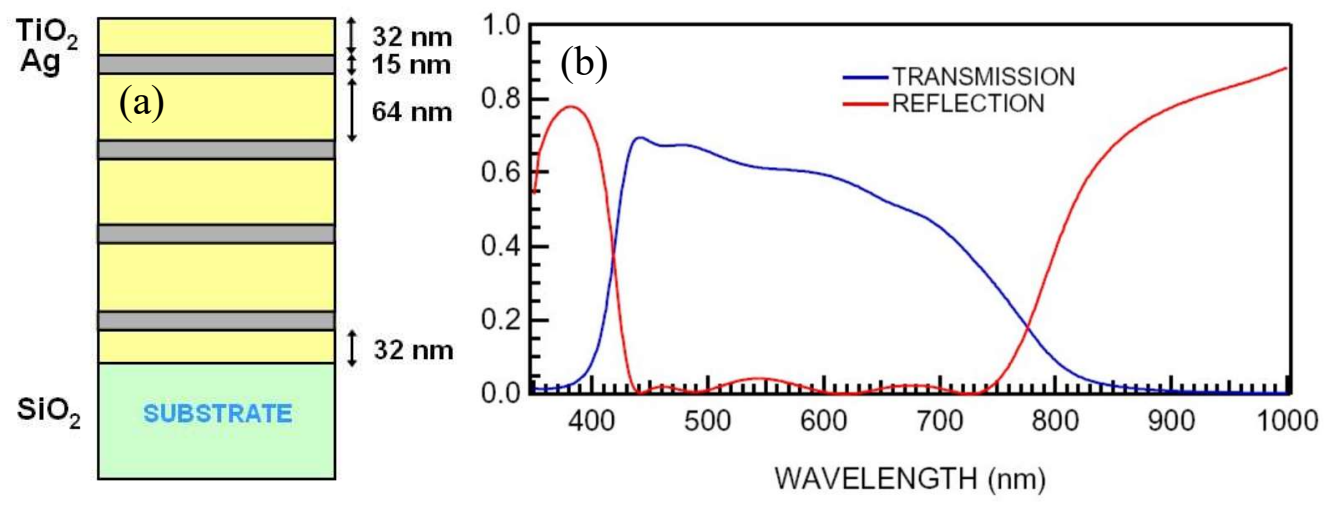

Fig. 17: (a) Diagram of the 'transparent-metal' photonic crystal sample. (b) Calculated reflection and transmission spectra of this sample.

Figure 17 (b) shows the calculated optical reflection and transmission spectra. In spite of the total thickness of $\mathrm{Ag}$ in the sample being $120 \mathrm{~nm}$, the sample has a surprisingly high transmission in a band around 400 to $700 \mathrm{~nm}$, hence the naming as a transparentmetal structure. The experimental transmission spectrum was measured, and found to be nearly identical to that calculated.

Picosecond laser ultrasonics experiments were carried out using 200 fs pump light pulses of wavelength $800 \mathrm{~nm}$.[54] The pump light is absorbed in the Ag films, and leads to a transient strain distribution in the sample. This transient distribution can be calculated using the transfer matrix method considered above for the optical absorption of the pump light together with a stress generation and propagation theory similar to that described in Section 2.[8] Figure 18 shows a simulation of the strain distribution 50 ps and $100 \mathrm{ps}$ after the arrival of the pump pulse at normal incidence from the top of the sample, taking optical and mechanical constants from the literature. The ultrasonic attenuation in the $\mathrm{TiO}_{2}$ films (varying quadratically with the acoustic frequency) is also taken into account. The shape of the strain pulse changes rapidly with time inside the films owing to multiple acoustic reflections. 

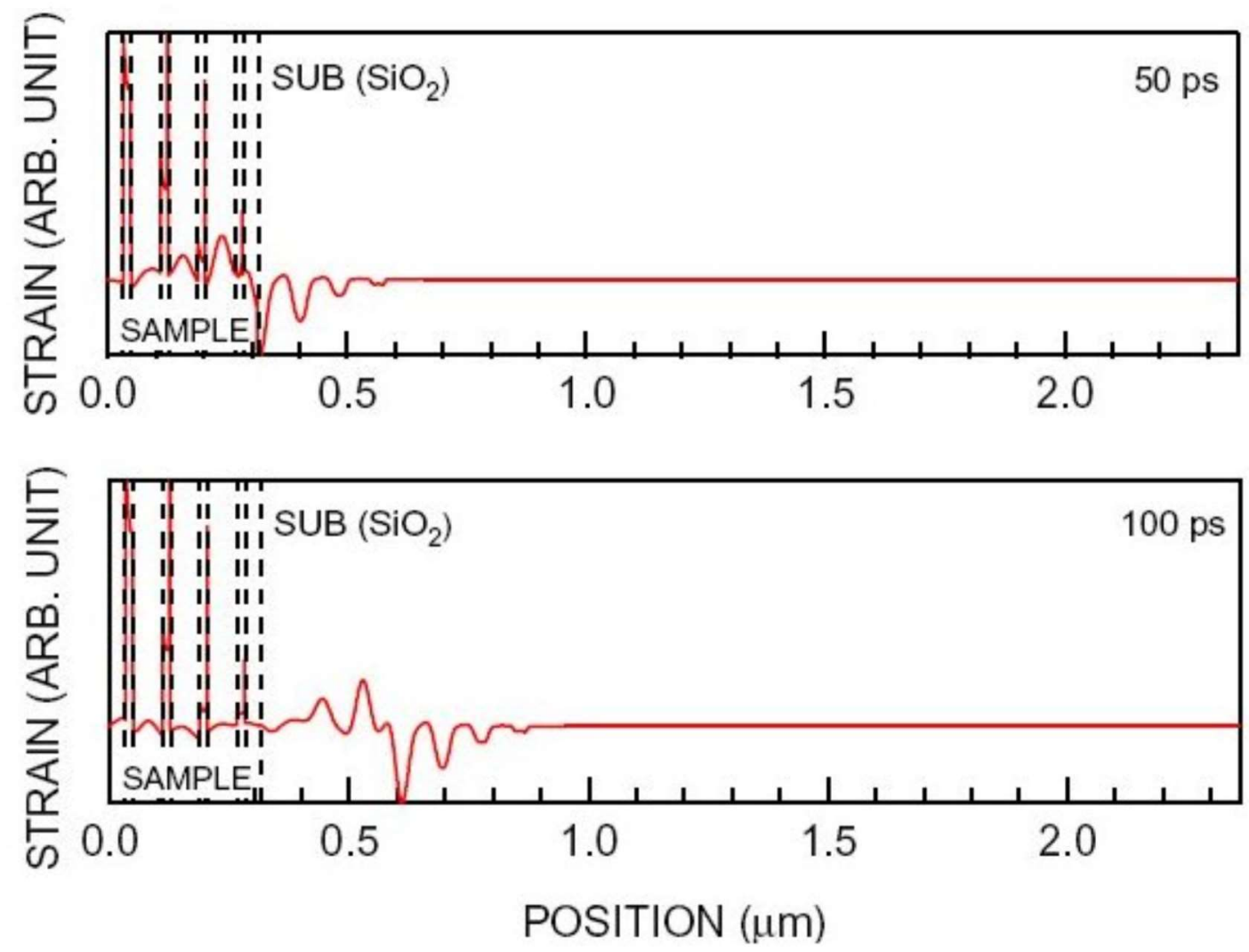

Fig. 18: Calculated strain distribution in the 'transparent-metal' sample 50 ps and $100 \mathrm{ps}$ after arrival of a $800 \mathrm{~nm}$ pump optical pulse from the top side of the sample at normal incidence. SUB means substrate.

Figure 19 shows typical results for the reflectivity $\delta R(t)$ using probe pulses of duration $200 \mathrm{fs}$ and wavelength $430 \mathrm{~nm}$. Also shown is a theoretical simulation based on Eq. (31), using optimized photoelastic constants and other constants from the literature.[54] 


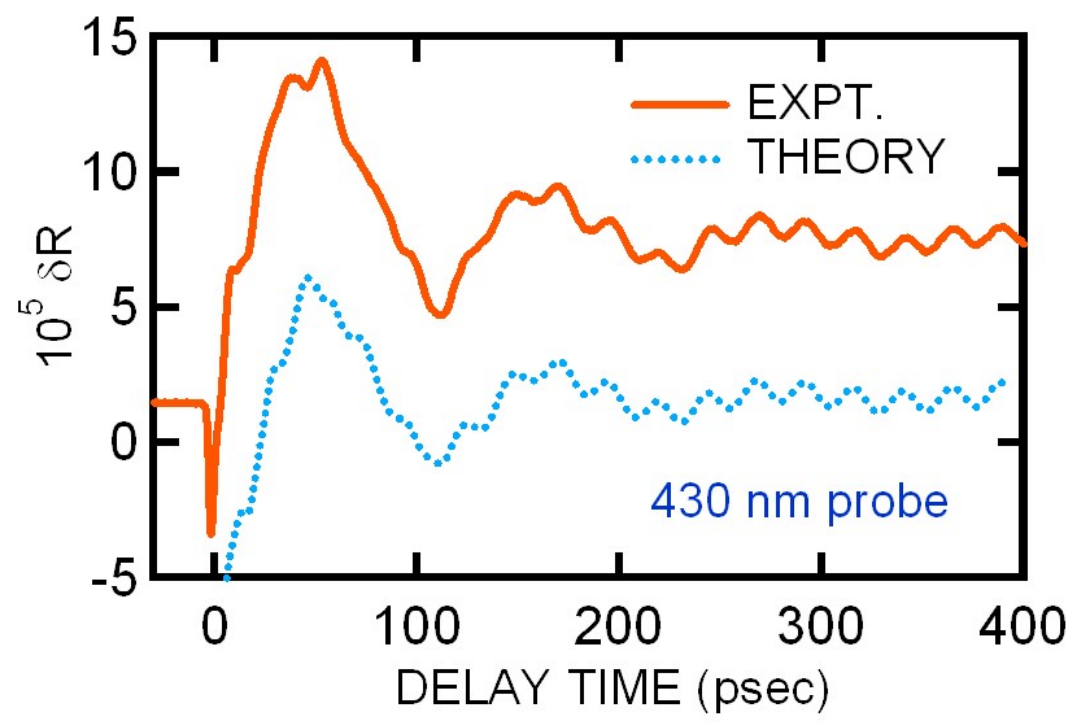

Fig. 19: Plot of the measured change in reflectivity as a function of delay time for the 'transparent-metal' sample using $800 \mathrm{~nm}$ pump optical pulses and $430 \mathrm{~nm}$ probe pulses. The optical pulses are incident on the top side of the sample at normal incidence. The theoretical predictions according to Eq. (35) are also shown by the dotted line.

Detailed analysis of the relative contributions to the signal shows that the high frequency oscillations in the signal are photoelastic in origin, whereas the lower frequency background signal arises from the interface displacements.

The idea of modulating a photonic crystal with picosecond ultrasonic waves may be of interest for the development of ultrafast acousto-optic modulators. It has also been studied, for example, in opal structures.[55,56] The problem one faces is how to achieve an efficient optical modulation. As can be seen from the vertical scale in Fig. 19, the typical amplitude $\delta R(t) \sim 10^{-4}$ is too small for useful applications. We found that matters are not much improved at other probe wavelengths in the range 390-430 nm investigated. However, it may be possible in the future to generate very high frequency ultrasonic waves electrically with much higher amplitudes,[57] thus facilitating the design of such high frequency acousto-optic modulators. 


\section{Detection at oblique optical incidence}

\section{The wave equation}

So far we have only dealt with normally-incident probe light, but in many experiments it is very useful to be able to work at a general angle of incidence. This presents advantages such as the ability to probe anisotropic materials effectively, to measure shear strain pulses, or to allow the separation of photoelastic and displacement contributions to the detected signals. The aim in this section is to analyse the case of oblique optical incidence in picosecond laser ultrasonics.[21, 24, 45, 58]

Our starting point is again the Maxwell equations, discussed in Section 2. We first consider an isotropic solid with a plane interface with a vacuum. With oblique optical incidence, Eq. (24) for the spatially dependent part of the electric field is generalized to

$$
\left[\left(\begin{array}{ccc}
\nabla^{2} & 0 & 0 \\
0 & \nabla^{2} & 0 \\
0 & 0 & \nabla^{2}
\end{array}\right)-\left(\begin{array}{ccc}
\partial^{2} / \partial x^{2} & \partial^{2} / \partial x \partial y & \partial^{2} / \partial x \partial z \\
\partial^{2} / \partial y \partial x & \partial^{2} / \partial y^{2} & \partial^{2} / \partial y \partial z \\
\partial^{2} / \partial \mathrm{z} \partial x & \partial^{2} / \partial \mathrm{z} \partial y & \partial^{2} / \partial z^{2}
\end{array}\right)+k^{2} \widetilde{\varepsilon}(z)\right] \mathbf{E}(\mathbf{r})=0
$$

where $\tilde{\varepsilon}(z)=\left(\varepsilon_{i j}\right)$ is the $3 \times 3$ complex relative permittivity tensor and $k=\omega \sqrt{\varepsilon_{0} \mu_{0}}$ is the vacuum wave number. Since the unperturbed solid is isotropic, and since perturbations in $\tilde{\varepsilon}$ do not break the lateral symmetry, we may without loss of generality always choose the directions of $x$ and $y$ so that $k_{y}=0, \partial / \partial y=\partial^{2} / \partial y^{2}=0$, and $\mathbf{E}=\mathbf{E}(z) \exp \left(i k_{x} x\right)$. Here the value of $k_{x}$ is imposed by the optical incidence conditions and is a known quantity. The wave equation then simplifies to

where

$$
\left[L\left(k_{x}\right)+k^{2} \tilde{\varepsilon}(z)\right] \mathbf{E}(z)=0,
$$

$$
L\left(k_{x}\right)=\left(\begin{array}{ccc}
\partial^{2} / \partial z^{2} & 0 & -i k_{x} \partial / \partial z \\
0 & \partial^{2} / \partial z^{2}-k_{x}^{2} & 0 \\
-i k_{x} \partial / \partial z & 0 & -k_{x}^{2}
\end{array}\right) .
$$

\section{Solution for the unperturbed case}

Consider first the unperturbed solution to Eq. (34) at the plane boundary of a vacuum and an isotropic solid. In this case,

$$
\begin{aligned}
& \varepsilon_{x x}(z)=\varepsilon_{y y}(z)=\varepsilon_{z z}(z)=\varepsilon_{h}(z)=\left\{\begin{array}{ll}
1 & (z<0) \\
\varepsilon & (z>0)
\end{array},\right. \\
& \varepsilon_{i j}(z)=0 \text { for } i \neq j .
\end{aligned}
$$

This geometry is sketched in Fig. 20 (a). Since there is no spatial dependence of $\varepsilon_{h}(z)=\varepsilon$ in the region $z>0$, let us define $\mathbf{E}(z)=\mathbf{E}_{0}(z)=\mathbf{E}_{1} \exp \left(i k_{z} z\right)$ in this unperturbed case. Equation (34) has a solution for $k_{z}$ provided that 


$$
\left|\begin{array}{ccc}
\varepsilon k^{2}-k_{z}{ }^{2} & 0 & k_{x} k_{z} \\
0 & \varepsilon k^{2}-k_{x}^{2}-k_{z}^{2} & 0 \\
k_{x} k_{z} & 0 & \varepsilon k^{2}-k_{x}^{2}
\end{array}\right|=0 .
$$

This is a fourth order equation in $k_{z}$ with four solutions, shown schematically in Fig. 20 (b). (The values of $k_{z}$ are complex quantities in general for complex $\varepsilon$.) The $z$ component of these wave vectors are labelled by $k_{j z}$, where $j=1, \cdots, 4$. So, in general (i.e. in the air or in the dielectric),

$$
\mathbf{E}=\sum_{j=1}^{4} a_{j} \mathbf{e}_{j} \exp \left(i k_{j z} z+i k_{x} x\right) .
$$

The reason why $k_{x}$ is not indexed is that the boundary conditions require that $k_{x}$ is a conserved quantity.
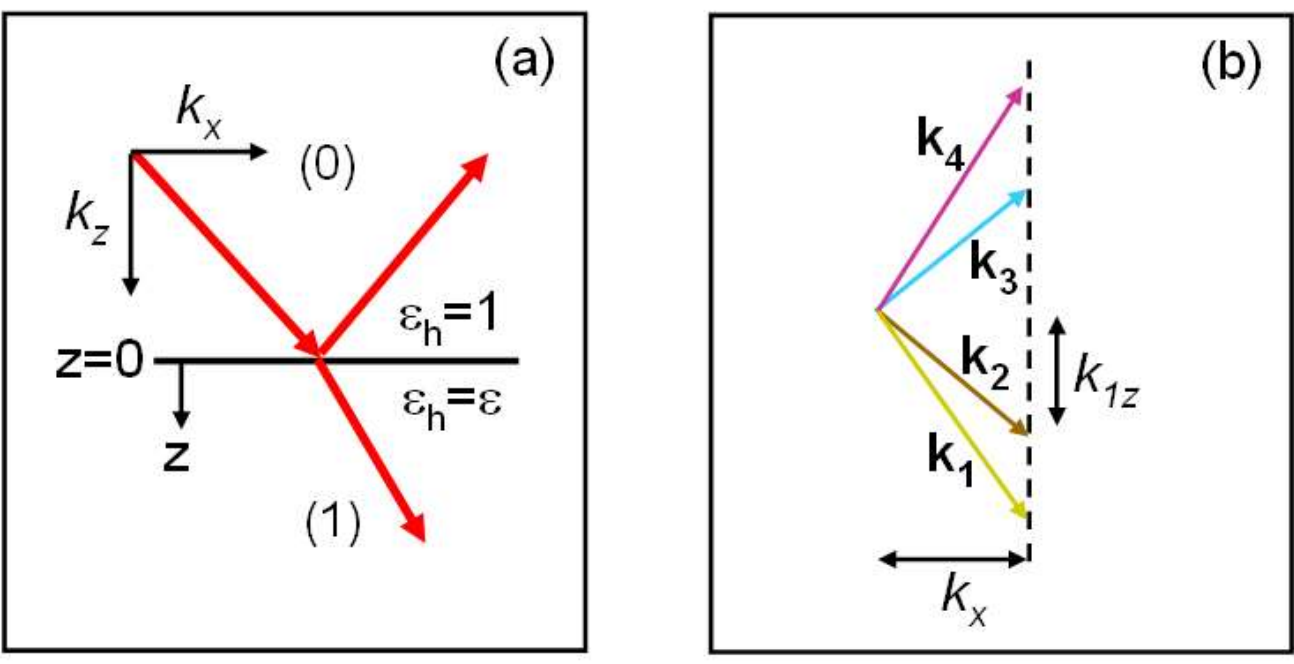

Fig. 20: (a) Geometry for optical incidence on an isotropic solid with a plane interface from a vacuum. (b) The general solution of the wave equation for this problem has four wave vectors. The horizontal component $k_{x}$, parallel to the interface, is a conserved quantity.

The general solutions for $k_{j z}$ are given in the vacuum (medium 0 ), by

$$
k_{1 z}{ }^{(0)}=k_{2 z}{ }^{(0)}=k^{\prime}, k_{3 z}{ }^{(0)}=k_{4 z}{ }^{(0)}=-k^{\prime},
$$

and in the dielectric (medium 1), by

$$
k_{1 z}^{(1)}=k_{2 z}^{(1)}=k^{\prime \prime}, k_{3 z}^{(1)}=k_{4 z}^{(1)}=-k^{\prime \prime},
$$

where $k^{\prime}=\sqrt{k^{2}-k_{x}{ }^{2}}$ and $k^{\prime \prime}=\sqrt{\varepsilon k^{2}-k_{x}{ }^{2}}$. We see immediately that the choice of $k_{x}$ has constrained the angle of optical incidence $\theta$ in the vacuum to $\tan \theta=k_{x} / k^{\prime}$. Each of these values of $k_{z}$, when substituted in Eq. (33), give the associated electric polarizations in Eq. (35). The result is 


$$
\begin{aligned}
& \mathbf{e}_{1}^{(0)}=\left(\begin{array}{l}
0 \\
1 \\
0
\end{array}\right), \mathbf{e}_{2}^{(0)}=\frac{1}{k}\left(\begin{array}{c}
k^{\prime} \\
0 \\
-k_{x}
\end{array}\right), \mathbf{e}_{3}^{(0)}=\mathbf{e}_{1}^{(0)}, \mathbf{e}_{4}^{(0)}=\frac{1}{k}\left(\begin{array}{c}
-k^{\prime} \\
0 \\
-k_{x}
\end{array}\right), \\
& \mathbf{e}_{1}^{(1)}=\left(\begin{array}{l}
0 \\
1 \\
0
\end{array}\right), \mathbf{e}_{2}^{(1)}=\frac{1}{k \sqrt{\varepsilon}}\left(\begin{array}{c}
k^{\prime \prime} \\
0 \\
-k_{x}
\end{array}\right), \mathbf{e}_{3}^{(1)}=\mathbf{e}_{1}^{(1)}, \mathbf{e}_{4}^{(1)}=\frac{1}{k \sqrt{\varepsilon}}\left(\begin{array}{c}
-k^{\prime \prime} \\
0 \\
-k_{x}
\end{array}\right) .
\end{aligned}
$$

Solving for the electric fields in the two media is now a matter of applying the boundary conditions on the fields. These can conveniently be expressed in matrix form

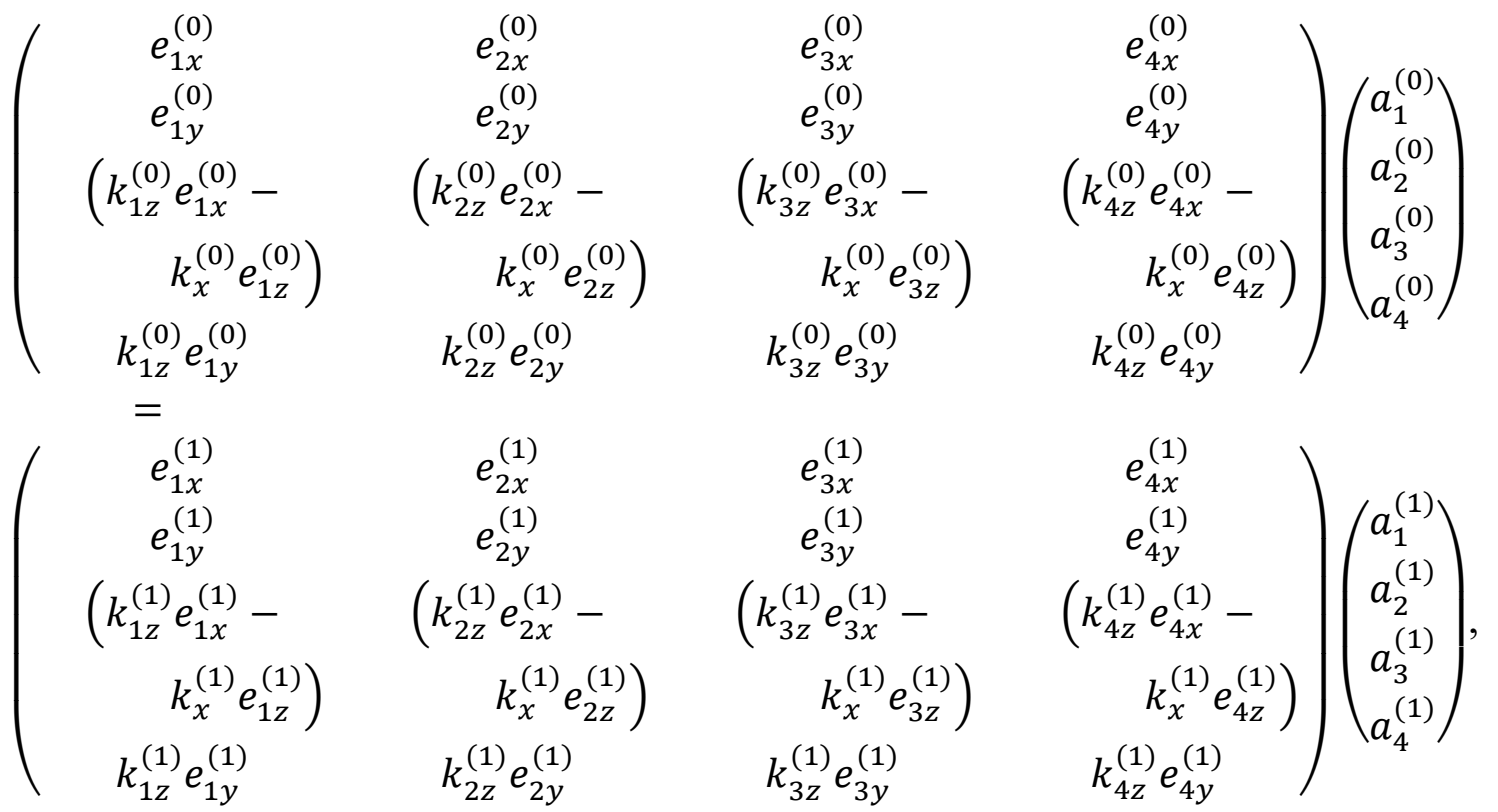

where, from top to bottom, the continuity of $E_{x}, E_{y}, D_{z}$ (equivalent to $B_{y}$ ) and $B_{x}$ have been used at $z=0$. A short form of this equation is $\mathbf{A}_{0} \mathbf{a}_{\mathbf{0}}=\mathbf{A}_{1} \mathbf{a}_{1}$. Setting $\mathbf{a}_{\mathbf{0}}=$ $\left(a_{1}, a_{2}, a_{1} r_{s}, a_{2} r_{p}\right)$ and $\mathbf{a}_{1}=\left(a_{1} t_{s}, a_{2} t_{p}, 0,0\right)$, where $a_{1}$ is the $s$-polarized component of the incident electric field and $a_{2}$ is the $p$-polarized component, the relation $\mathbf{a}_{\mathbf{0}}=$ $\mathbf{A}_{0}{ }^{-1} \mathbf{A}_{1} \mathbf{a}_{1}$ allows us to solve for the amplitude reflection coefficients $r_{s}, r_{p}$ and transmission coefficients $t_{s}, t_{p}$ :

$$
\begin{aligned}
& r_{s}=\frac{k^{\prime}-k^{\prime \prime}}{k^{\prime}+k^{\prime \prime}}, \\
& r_{p}=\frac{\varepsilon k^{\prime}-k^{\prime \prime}}{\varepsilon k^{\prime}+k^{\prime \prime}}, \\
& t_{s}=\frac{2 k^{\prime}}{k^{\prime}+k^{\prime \prime}}, \\
& t_{p}=\frac{2 \sqrt{\varepsilon} k^{\prime}}{\varepsilon k^{\prime}+k^{\prime \prime}} .
\end{aligned}
$$

As a by-product of our efforts we have succeeded in deriving the Fresnel equations for oblique reflection from a medium with a complex permittivity.

Obtaining the total fields from Eq. (35) is now straightforward as all the e and a 
vectors are known. Setting $a_{1}=1$ and $a_{2}=0$, for $s$-polarization we find

$$
\mathbf{E}_{0}(z)=\left\{\begin{array}{cc}
\left(e^{i k^{\prime} z}+r_{s} e^{-i k^{\prime} z}\right)\left(\begin{array}{l}
0 \\
1 \\
0
\end{array}\right) & \text { for } z<0, \\
t_{s} e^{i k^{\prime \prime} z}\left(\begin{array}{l}
0 \\
1 \\
0
\end{array}\right) & \text { for } z>0,
\end{array}\right.
$$

whereas setting $a_{1}=0$ and $a_{2}=1$ for $p$-polarization,

$$
\mathbf{E}_{0}(z)=\left\{\begin{array}{c}
\frac{e^{i k^{\prime} z}}{k}\left(\begin{array}{c}
k^{\prime} \\
0 \\
-k_{x}
\end{array}\right)+r_{p} \frac{e^{-i k^{\prime} z}}{k}\left(\begin{array}{c}
-k^{\prime} \\
0 \\
-k_{x}
\end{array}\right) \text { for } z<0, \\
t_{p} \frac{e^{i k^{\prime \prime} z}}{k \sqrt{\varepsilon}}\left(\begin{array}{c}
k^{\prime \prime} \\
0 \\
-k_{x}
\end{array}\right) \text { for } z>0 .
\end{array}\right.
$$

\section{Solution for the perturbed case}

The equation (33) to be solved is

$$
\left[L\left(k_{x}\right)+k^{2}\left(\tilde{\varepsilon}_{h}(z)+\tilde{\varepsilon}_{i h}(z)\right)\right] \mathbf{E}(z)=0,
$$

where, as in Section 2, we have again split $\tilde{\varepsilon}(z)$ into homogeneous and inhomogeneous parts. This equation can be solved with the help of a $3 \times 3$ Green's matrix $G\left(z, z^{\prime}\right)$ that satisfies

$$
\left[L\left(k_{x}\right)+k^{2} \tilde{\varepsilon}_{h}(z)\right] G\left(z, z^{\prime}\right)=-\mathbf{I} \delta\left(z-z^{\prime}\right),
$$

together with the above solutions for $\mathbf{E}_{0}(z)$ to the equation

$$
\left[L\left(k_{x}\right)+k^{2} \tilde{\varepsilon}_{h}(z)\right] \mathbf{E}_{0}(z)=0,
$$

where $\mathbf{I}$ is the $3 \times 3$ identity matrix.

The perturbation to the permittivity can be written $\tilde{\varepsilon}_{i h}=\tilde{\varepsilon}_{p e}+\tilde{\varepsilon}_{d}$, where $\tilde{\varepsilon}_{p e}$ is caused by the photoelastic effect and $\tilde{\varepsilon}_{d}$ by the displacement of the surface. From Eq. (26), we have

$$
\tilde{\varepsilon}_{p e}(z)=\eta(z)\left(\begin{array}{ccc}
P_{12} & 0 & 0 \\
0 & P_{12} & 0 \\
0 & 0 & P_{11}
\end{array}\right)
$$

where, as before, $\eta$ is the longitudinal component of strain $\left(\eta=\eta_{z z}\right.$, all other components being zero). From Eq. (27), we have, for the $x x, y y$, and $z z$ components of $\tilde{\varepsilon}_{d}$, 


$$
\tilde{\varepsilon}_{d}=\left\{\begin{aligned}
(1-\varepsilon) \mathbf{I} & \text { for } u>0,0<z<u, \\
(\varepsilon-1) \mathbf{I} & \text { for } u<0, u<z<0, \\
0 \quad \text { elsewhere. } &
\end{aligned}\right.
$$

(This represents the top hat distribution of Fig. 16 at the surface, which is drawn for the case of $u>0$ at the 1-2 interface.) With identical reasoning to that leading to Eq. (31), we may write

$$
\begin{aligned}
\mathbf{E}(z) \cong \mathbf{E}_{0}(z)+k^{2} \int_{-\infty}^{\infty} G\left(z, z^{\prime}\right) \tilde{\varepsilon}_{i h}\left(z^{\prime}\right) \mathbf{E}_{0}\left(z^{\prime}\right) d z^{\prime} \\
\cong \mathbf{E}_{0}(z)+k^{2} \int_{-\infty}^{\infty} G\left(z, z^{\prime}\right) \tilde{\varepsilon}_{p e}\left(z^{\prime}\right) \mathbf{E}_{0}\left(z^{\prime}\right) d z^{\prime}+k^{2}(1-
\end{aligned}
$$

ع) $u G(z,-0) \mathbf{E}_{0}(+0)$,

where the last term in Eq. (39) has been specifically written for the case of outward motion $(u<0)$ in which part of medium 1 penetrates into the region of medium 0 . The values for $z$ just greater and less than zero $(z=+0$ and $z=-0)$ should be distinguished because of discontinuities in the field $E_{z}$ and in the Green's function $G$ at the interface, a problem that does not arise for normal incidence: the choice of $\mathbf{E}_{0}(+0)$ ensures the continuity in the $z$ component of $\mathbf{E}_{0}$ at $z=0$ because medium 1 is present at this point if $u<0$; in addition, the choice of $G(z,-0)$ is a result of $G\left(z, z^{\prime}\right)$ being integrated over $z^{\prime}$ in medium 0 , as it should be for $u<0$ (since the integral should be evaluated with $G$ obtained under the same conditions as the unperturbed problem for which $u=0)$. In the case $u>0$ one should use $\mathbf{E}_{0}(-0)$ and $G(z,+0)$ instead. It turns out, however, that $G(z,+0) \mathbf{E}_{0}(-0)=G(z,-0) \mathbf{E}_{0}(+0)$ and thus Eq. (39) can be used for any $u$ regardless of its polarity.

The required Green's matrices are given by

$$
\begin{aligned}
& G\left(z, z^{\prime} ; k_{x}\right)= \\
& \left(\begin{array}{ccc}
\frac{i k^{\prime} k^{\prime \prime}}{k^{2}\left(k^{\prime \prime}+\varepsilon k^{\prime}\right)} & 0 & \frac{i k^{\prime} k_{x}}{k^{2}\left(k^{\prime \prime}+\varepsilon k^{\prime}\right)} \\
0 & \frac{i}{k^{\prime}+k^{\prime \prime}} & 0 \\
\frac{i k^{\prime \prime} k_{x}}{k^{2}\left(k^{\prime \prime}+\varepsilon k^{\prime}\right)} & 0 & \frac{i k_{x}^{2}}{k^{2}\left(k^{\prime \prime}+\varepsilon k^{\prime}\right)}
\end{array}\right) e^{i\left(k^{\prime \prime} z^{\prime}-k^{\prime} z\right)} \text { for } \mathrm{z}<0, z^{\prime}>0 \text {, } \\
& \left(\begin{array}{ccc}
\frac{i k^{\prime}\left(k^{\prime \prime}-\varepsilon k^{\prime}\right)}{2 k^{2}\left(k^{\prime \prime}+\varepsilon k^{\prime}\right)} & 0 & \frac{-i k_{x}\left(k^{\prime \prime}-\varepsilon k^{\prime}\right)}{2 k^{2}\left(k^{\prime \prime}+\varepsilon k^{\prime}\right)} \\
0 & \frac{i\left(k^{\prime}-k^{\prime \prime}\right)}{2 k^{\prime}\left(k^{\prime}+k^{\prime \prime}\right)} & 0 \\
\frac{i k_{x}\left(k^{\prime \prime}-\varepsilon k^{\prime}\right)}{2 k^{2}\left(k^{\prime \prime}+\varepsilon k^{\prime}\right)} & 0 & \frac{-i k_{x}^{2}\left(k^{\prime \prime}-\varepsilon k^{\prime}\right)}{2 k^{2} k^{\prime}\left(k^{\prime \prime}+\varepsilon k^{\prime}\right)}
\end{array}\right) e^{-i k^{\prime}\left(z^{\prime}+z\right)+} \\
& \left(\begin{array}{ccc}
\frac{i k^{\prime}}{2 k^{2}} & 0 & \frac{i k_{x}}{2 k^{2}} \operatorname{sgn}\left(z^{\prime}-z\right) \\
0 & \frac{i}{2 k^{\prime}} & 0 \\
\frac{i k_{x}}{2 k^{2}} \operatorname{sgn}\left(z^{\prime}-z\right) & 0 & \frac{i k_{x}^{2}}{2 k^{2} k^{\prime}}
\end{array}\right) e^{i k^{\prime}\left|z^{\prime}-z\right|}-\left(\begin{array}{ccc}
0 & 0 & 0 \\
0 & 0 & 0 \\
0 & 0 & 1
\end{array}\right) \frac{\delta\left(z^{\prime}-z\right)}{k^{2}}
\end{aligned}
$$




$$
\text { for } z<0, z^{\prime}<0 \text {. }
$$

These ranges of $z$ and $z^{\prime}$ suffice to solve the problem in hand. These Green's matrices are also discussed in Refs. [24] and [59]. The reciprocity relation $G\left(z, z^{\prime} ; k_{x}\right)=$ $G^{T}\left(z^{\prime}, z ;-k_{x}\right)$ can be shown to hold, where $T$ means the transpose of the matrix.

The next step is to substitute for $G$ into Eq. (39) at a point $z<0$. The result for $s$ polarization, including time dependence, is

$$
\begin{gathered}
\frac{\delta r_{S}(t)}{r_{0}}=\frac{2 i k^{\prime} k^{2} P_{12}}{\left(k^{\prime}-k^{\prime \prime}\right)\left(k^{\prime}+k^{\prime \prime}\right)} \int_{0}^{\infty} \eta\left(z^{\prime}, t\right) e^{2 i k^{\prime \prime} z^{\prime}} d z^{\prime}+2 i k^{\prime} u(t) \\
=\frac{2 i k^{\prime} P_{12}}{1-\varepsilon} \int_{0}^{\infty} \eta\left(z^{\prime}, t\right) e^{2 i k^{\prime \prime} z^{\prime}} d z^{\prime}+2 i k^{\prime} u(t),
\end{gathered}
$$

and the result for $p$-polarization is

$$
\frac{\delta r_{p}(t)}{r_{0}}=\frac{2 i k^{\prime}\left(k^{\prime \prime 2} P_{12}-k_{x}^{2} P_{11}\right)}{{k^{\prime \prime}}^{2}-\varepsilon^{2}{k^{\prime}}^{2}} \int_{0}^{\infty} \eta\left(z^{\prime}, t\right) e^{2 i k^{\prime \prime} z^{\prime}} d z^{\prime}+2 i k^{\prime} u(t)
$$

where the components of $k_{z}, k^{\prime}=\sqrt{k^{2}-k_{x}{ }^{2}}$ and $k^{\prime \prime}=\sqrt{\varepsilon k^{2}-k_{x}{ }^{2}}$, are determined by $k_{x}, \varepsilon$ and $k=\omega / c$. Equations (40) and (41) can be shown to be valid for either sign of $u$. At normal incidence, for which $k_{x}=0, k^{\prime}=k$, and $k^{\prime}=k \sqrt{\varepsilon}$, both of these equations reduce to

$$
\frac{\delta r(t)}{r_{0}}=\frac{2 i k P_{12}}{1-\varepsilon} \int_{0}^{\infty} \eta\left(z^{\prime}, t\right) e^{2 i \sqrt{\varepsilon} k z^{\prime}} d z^{\prime}+2 i k u(t)
$$

Given that $P_{12}=2 \tilde{n} d \tilde{n} / d \eta$, this agrees with Eq. (9). The equation for $\delta r_{p} / r_{0}$ involves both photoelastic constants $P_{12}$ and $P_{11}$ because $\mathbf{E}$ has both $x$ and $z$ components, whereas the equation for $\delta r_{s} / r_{0}$, involves only $P_{12}$ because in this case $\mathbf{E}$ has only a $y$ component. Both equations have the same integral term as Eq. (9), but the difference lies in the complex prefactor and the wave number in the exponential that affect the time dependence of the observed signals (i.e. $\rho$ or $\delta \phi$ ). The response to a strain pulse being reflected from the free surface inside the solid is thus qualitatively similar to the case for normal incidence. The frequency of the oscillations in the oscillatory term is, however, reduced to the value $f_{n}=2 n v_{l} \cos \theta / \lambda$, where $\theta$ is the angle of optical incidence.

Experiments on isotropic solids with oblique probe incidence allow us extra degrees of freedom. It has been suggested, for example, that by measuring both $\delta r_{s} / r_{0}$ and $\delta r_{p} / r_{0}$ one should be able to experimentally separate the photoelastic and surface displacement contributions.[45] The suggested method has been implemented as a interferometric setup to observe the surface displacement directly without contamination by the photoelastic effect.[46] Resolving the surface displacement contribution $\propto u(t)$ has the advantage of directly probing the shape (i.e. the integral) of the strain pulse in the solid. This allows ultrafast diffusion processes to be investigated accurately. Another useful application is in the tomographic reconstruction of picosecond acoustic strain propagation by scanning the angle of probe incidence $\theta$, in which a modified oblique-incidence theory is used to account for incidence from a medium with a relative permittivity different from unity.[60] 
For arbitrarily cut anisotropic materials the allowed acoustic modes for a general propagation direction have in general both longitudinal and shear strain components. It is possible to generate these shear components as a consequence of the broken symmetry. The thermal expansion tensor does not have to be anisotropic for this to occur. If shear components are present one has to deal with off-diagonal elements in the perturbed permittivity tensor. Without going into great detail we will briefly review this problem with relation to picosecond shear pulse detection.[21, 58] A general treatment of transient light scattering including shear strain detection has been given in Ref. [24] based on Green's functions. Another theoretical description of shear strain detection using a Jones matrix formalism has been given in Ref. [61].

Consider as an example an anisotropic opaque substrate coated with a transparent isotropic film. This facilitates the analysis of the photoelastic detection process in the isotropic film, which supports only pure shear or pure longitudinal modes (as opposed to quasilongitudinal or quasishear modes of mixed polarization). In general, a $z$ propagating strain pulse in the isotropic film (originating from optical excitation of the opaque substrate) may have non-zero shear strain components $\eta_{4}=2 \eta_{y z}$ and $\eta_{5}=$ $2 \eta_{x z}$, and a longitudinal component $\eta_{3}=\eta_{z z}$. These can modulate the relative permittivity tensor of the film according to the photoelastic effect through $\Delta \varepsilon_{I}=P_{I J} \eta_{J}$ [see discussion after Eq. (26)], an equation that now includes off-diagonal components. With the same notation as Section 2 and 3, and taking the photoelastic tensor for an isotropic solid,[38]

$$
\begin{gathered}
\tilde{\varepsilon}_{p e}=\left(\begin{array}{ccc}
P_{12} \eta_{3} & 0 & P_{44} \eta_{5} \\
0 & P_{12} \eta_{3} & P_{44} \eta_{4} \\
P_{44} \eta_{5} & P_{44} \eta_{4} & P_{11} \eta_{3}
\end{array}\right) \\
=\left(\begin{array}{ccc}
P_{12} \eta_{3} & 0 & 0 \\
0 & P_{12} \eta_{3} & 0 \\
0 & 0 & P_{11} \eta_{3}
\end{array}\right)+P_{44}\left(\begin{array}{ccc}
0 & 0 & \eta_{5} \\
0 & 0 & \eta_{4} \\
\eta_{5} & \eta_{4} & 0
\end{array}\right),
\end{gathered}
$$

where we have used $\varepsilon_{1}=\varepsilon_{x x}, \varepsilon_{4}=2 \varepsilon_{x z}$ etc., and $P_{11}, P_{12}$, and $P_{44}=\left(P_{11}-P_{12}\right) / 2$ are the photoelastic tensor components for an isotropic material (as explained in Section 2). Since the coupling of $\tilde{\varepsilon}$ to shear strain $\eta_{y z}$ or $\eta_{x z}$, [the second term in Eq. (42) ] is off-diagonal and involves the suffix $z$, it will only couple to the $z$ component of the probe light electric field [as can be seen from Eq. (34)]. Therefore, $p$-polarized probe light must be involved (either in incidence or in reflection) to detect shear waves in isotropic materials under oblique incidence conditions.

We can use Eq. (39) to calculate the perturbed electric fields corresponding to the shear strain assuming, as before, that the $x$ axis lies within the plane of incidence. For $s$ polarization, we obtain

$$
\mathbf{E}(z) \cong \mathbf{E}_{0}(z)-\frac{2 i k^{\prime} k_{x} P_{44} e^{-i k^{\prime} z}}{\left(k^{\prime}+k^{\prime \prime}\right)\left(\varepsilon k^{\prime}+k^{\prime \prime}\right)} \frac{1}{k}\left(\begin{array}{c}
-k^{\prime} \\
0 \\
-k_{x}
\end{array}\right) \int_{0}^{\infty} \eta_{4}\left(z^{\prime}\right) e^{2 i k^{\prime \prime} z^{\prime}} d z^{\prime}
$$

Whereas for $p$-polarization, we obtain 


$$
\mathbf{E}(z) \cong \mathbf{E}_{0}(z)-\frac{2 i k k^{\prime} k_{x} P_{44} e^{-i k^{\prime} z}}{\left(k^{\prime}+k^{\prime \prime}\right)\left(\varepsilon k^{\prime}+k^{\prime \prime}\right)}\left(\begin{array}{l}
0 \\
1 \\
0
\end{array}\right) \int_{0}^{\infty} \eta_{4}\left(z^{\prime}\right) e^{2 i k^{\prime \prime} z^{\prime}} d z^{\prime}
$$

where Eqs. (36) and (37) for $z<0$ respectively determine $\mathbf{E}_{0}(z)$ in Eqs. (43) and (44). These equations imply the following,: (i) only $\eta_{4}$ shear strain can be detected; (ii) the strain $\eta_{4}$ scatters $s$-polarized to $p$-polarized light and vice versa, as shown in Fig. 21; (iii) a first order change in $R\left(\delta R \propto \eta_{4}\right)$ can be obtained only by allowing the incident and reflected light to interfere (otherwise $\delta R \propto \eta_{4}{ }^{2}$ ). This interference is straightforward to arrange by mixing a portion of the incident probe light with the scattered light. In the case of plane shear waves reflecting at normal incidence from a free surface in an isotropic medium, there is no normal surface displacement, and so we do not have to contend with the contribution due to $\tilde{\varepsilon}_{d}$ in this case.
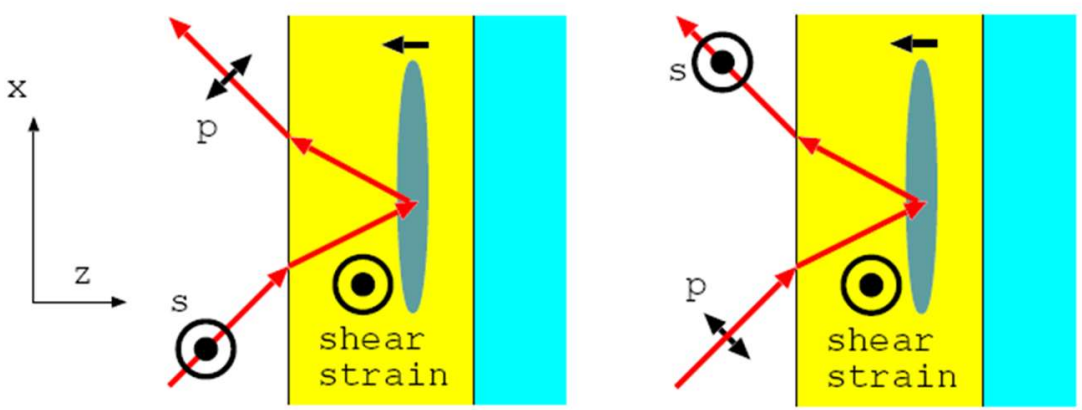

Fig. 21: Schematic diagram showing how a shear strain $\eta_{4}=2 \eta_{y z}$ couples $s$ polarized light to $p$-polarized light and vice versa. This example shows a shear pulse returning to the surface of a thin film on a substrate.

The analysis for optical strain detection in an anisotropic substrate is more complex. In general the shear strain can perturb both the diagonal and off-diagonal components of the permittivity tensor. Therefore there are no strict conditions on the choice of polarization for the probe light for the detection of shear strain in this case. For anisotropic media, an extra contribution to the perturbation of the permittivity arises because of induced local rotations of the solid that accompany any strain. This rotation contribution must also be taken into account in the theory. In addition, acoustic mode conversion at the interface needs to be accounted for. A general analysis method incorporating all these effects has been discussed using the Green's function for an arbitrary anisotropic multilayer system.[24]

The detection of shear strain was experimentally verified in a variety of systems.[21, 62] Shear strain arising from 3D propagation effects has also been detected.[25, 63] Shear strain wave propagation in liquids has also been studied, following on from similar experiments for longitudinal waves in mercury.[64, 65] One can expect more interesting developments in this field.

\section{Conclusions}

In conclusion, this paper reviews the fundamentals of the generation and detection of picosecond strain pulses in solids. We explained in detail the shape of the acoustic echoes in both amplitude and phase for generation at the free surface of an opaque 
isotropic solid and for detection with normally-incident optical probe pulses. We extended the detection theory to isotropic multilayers using a Green's function method, and applied this approach to a photonic crystal. The detection theory was further extended to handle probe light at oblique incidence, and this theory was applied to the detection of shear waves. Not everything could be covered in this article. For example, one area of rapid development is the field of nonlinear picosecond laser ultrasonics, involving the generation and detection of picosecond acoustic solitons or shock waves in crystalline solids using ultrashort light pulses.[66-70] Work in picosecond laser ultrasonics is progressing rapidly, and we can look forward to fascinating developments in this field.

\section{Acknowledgements}

We thank Vitali Gusev, Bernard Perrin and Motonobu Tomoda for stimulating discussions on this subject, and Jeremy Baumberg for suggesting the two-dimensional echo plots. We also thank Eitaro Mizunuma for experimental support and Francesca Sarto for sample growth.

\section{References}

[1] C. Thomsen, H. T. Grahn, H. J. Maris, and J. Tauc, Phys. Rev. B 34, 4129 (1986).

[2] O. B. Wright and K. Kawashima, Phys. Rev. Lett. 69, 1668 (1992).

[3] S. A. Akmanov and V. E. Gusev, Sov. Phys. Usp. 35, 153 (1992).

[4] V. E. Gusev and A. A. Karabutov, Laser Optoacoustics (American Institute of Physics, Woodbury, NY, 1993).

[5] B. Perrin, in Microscale and Nanoscale Heat Transfer, ed. S. Volz, Topics in Appl. Physics, Vol. 107 (Springer Verlag, Berlin Heidelberg, 2007), p. 333.

[6] L. D. Landau and E. M. Lifshitz, Mechanics (Elsevier, New York, 1982).

[7] W. Nowacki, Thermoelasticity (Pergamon Press, Oxford, London, New York, Paris, 1962).

[8] O. Matsuda and O. B. Wright, J. Opt. Soc. Am. B 19, 3028 (2002).

[9] T. Saito, O. Matsuda, and O. B. Wright, Phys. Rev. B 67, 205421 (2003).

[10] T. Pezeril, V. Gusev, D. Mounier, N. Chigarev, and P. Ruello, J. Phys. D: Appl. Phys. 38, 1421 (2005).

[11] O. B. Wright, Phys. Rev. B 49, 9985 (1994).

[12] G. Tas and H. J. Maris, Phys. Rev. B 49, 15046 (1994).

[13] O. B. Wright and V. E. Gusev, IEEE Trans. Ultrason. Ferro. Freq. Cont. 42, 331 (1995).

[14] N. V. Chigarev, D. Y. Paraschuk, X. Y. Pan, and V. E. Gusev, Phys. Rev. B 61, 15837 (2000).

[15] O. B. Wright, B. Perrin, O. Matsuda, and V. E. Gusev, Phys. Rev. B 64, 081202 (2001).

[16] O. Matsuda, T. Tachizaki, T. Fukui, J. J. Baumberg, and O. B. Wright, Phys. Rev. B 71, 115330 (2005).

[17] O. B. Wright and V. E. Gusev, Appl. Phys. Lett. 66, 1190 (1995).

[18] G.-W. Chern, K.-H. Lin, and C.-K. Sun, J. Appl. Phys. 95, 1114 (2004).

[19] Ü. Ozgür, C.-W. Lee, and H. O. Everitt, Phys. Rev. Lett. 86, 5604 (2001).

[20] K.-H. Lin, G.-W. Chern, Y.-K. Huang, and C.-K. Sun, Phys. Rev. B 70, 073307 (2004). 
[21] O. Matsuda, O. B. Wright, D. H. Hurley, V. E. Gusev, and K. Shimizu, Phys. Rev. Lett. 93, 095501 (2004).

[22] Y.-C. Wen, L.-C. Chou, H.-H. Lin, V. Gusev, K.-H. Lin, and C.-K. Sun, Appl. Phys. Lett. 90, 172102 (2007).

[23] P. Babilotte, P. Ruello, G. Vaudel, T. Pezeril, D. Mounier, J.-M.Breteau, and V. Gusev, Appl. Phys. Lett. 97, 174103 (2010).

[24] O. Matsuda, O. B. Wright, D. H. Hurley, V. Gusev, and K. Shimizu, Phys. Rev. B 77, 224110 (2008).

[25] C. Rossignol, J. M. Rampnoux, M. Perton, B. Audoin, and S. Dilhaire, Phys. Rev. Lett. 94, 166106 (2005).

[26] O. B. Wright, J. Appl. Phys. 71, 1617 (1992).

[27] O. B. Wright, T. Hyoguchi, and K. Kawashima, Jpn. J. Appl. Phys. 31, 85 (1992).

[28] S. Kashiwada, O. Matsuda, J. J. Baumberg, R. Li Voti, and O. B. Wright, J. Appl. Phys. 100, 073506 (2006).

[29] A. Yamamoto, T. Mishina, Y. Masumoto, and M. Nakayama, Phys. Rev. Lett. 73, 740 (1994).

[30] P. Basséras, S. M. Gracewski, G. W. Wicks, and R. J. D. Miller, J. Appl. Phys. 75, 2761 (1994).

[31] K. Mizoguchi, M. Hase, S. Nakashima, and M. Nakayama, Phys. Rev. B 60, 8262 (1999).

[32] C.-K. Sun, J.-C. Liang, and X.-Y. Yu, Phys. Rev. Lett. 84, 179 (2000).

[33] J. S. Yahng, Y. D. Jho, K. J. Yee, E. Oh, J. C. Woo, D. S. Kim, G. D. Sanders, and C. J. Stanton, Appl. Phys. Lett. 80, 4723 (2002).

[34] N.-W. Pu, Phys. Rev. B 72, 115428 (2005).

[35] K.-H. Lin, C.-F. Chang, C.-C. Pan, J.-I. Chyi, S. Keller, U. Mishra, S. P. DenBaars, and C.-K. Sun, Appl. Phys. Lett. 89, 143103 (2006).

[36] A. V. Akimov, A. V. Scherbakov, D. R. Yakovlev, C. T. Foxon, and M. Bayer, Phys. Rev. Lett. 97, 037401 (2006).

[37] M. F. P. Winter, G. Rozas, A. Fainstein, B. Jusserand, B. Perrin, A. Huynh, P. O. Vaccaro, and S. Saravanan, Phys. Rev. Lett. 98, 265501 (2007).

[38] J. F. Nye, Physical Properties of Crystals (Oxford University Press, Oxford, UK, 1957).

[39] M. Grimsditch, "Brillioun Scattering", in Handbook of Elastic Properties of Solids, Liquids and Gases, Volume 1: Dynamic Methods for Measuring the Elastic Properties of Solids, eds. M. Levy, H. Bass, R. Stern, and V. Keppens. (Academic Press, New York, 2001), p. 331.

[40] A. Yariv and P. Yeh, Optical Waves in Crystals (Wiley, New York, 1984).

[41] H. N. Lin, R. J. Stoner, H. J. Maris, J. Tauc, and., J. Appl. Phys. 69, 3816 (1991).

[42] B. Perrin, B. Bonello, J.-C. Jeannet, and E. Romatet, Prog. Nat. Sci. S6, 444 (1996).

[43] D. H. Hurley and O. B. Wright, Opt. Lett. 24, 1305 (1999).

[44] T. Tachizaki, T. Muroya, O. Matsuda, Y. Sugawara, D. H. Hurley, and O. B. Wright, Rev. Sci. Instrum. 77, 043713 (2006).

[45] O. Matsuda and O. B. Wright, Rev. Sci. Instrum. 74, 895 (2003).

[46] O. Matsuda, M. Tomoda, T. Tachizaki, S. Koiwa, A. Ono, K. Aoke, R. P. Beardsley, and O. B. Wright, J. Opt. Soc. Am. B 30, 1911 (2013).

[47] O. B. Wright, Opt. Lett. 20, 632 (1995).

[48] V. E. Gusev, Acustica 82, S37 (1996).

[49] A. Feldman and R. M. Waxler, J. Appl. Phys. 53, 1477 (1982).

[50] F. A. Abelès, "Optics of thin films", in Advanced Optical Techniques, ed. A. C. S. van Heel, Chap. 5 (Wiley, New York, 1967), p. 143. 
[51] M. Born and E. Wolf, Principles of Optics, 7th ed. (Cambridge U. Press, Cambridge, UK, 1999).

[52] M. Scalora, M. J. Bloemer, A. S. Pethel, J. P. Dowling, C. M. Bowden, and A. S. Manka, J. Appl. Phys. 84, 2377 (1998).

[53] M. S. Sarto, R. Li Voti, F. Sarto, M. C. Larciprete, IEEE Trans. Electromagn. Compat. 47, 602 (2005). The sample was obtained from M. C. Larciprete.

[54] E. Mizunuma, Masters Thesis, Graduate School of Engineering, Division of Applied Physics, Hokkaido University (2005) (in Japanese).

[55] C. Mechri, P. Ruello, D. Mounier, J. M. Breteau, I. Povey, M. Pemble, S. G. Romanov, and V. Gusev, J. Phys: Conf. Ser. 92, 012030 (2007).

[56] A. V. Akimov, Y. Tanaka, A. B. Pevtsov, S. F. Kaplan, V. G. Golubev, S. Tamura, D. R. Yakovlev, and M. Bayer, Phys. Rev. Lett. 101, 033902 (2008).

[57] A. J. Kent, R. N. Kini, N. M. Stanton, M. Henini, B. A. Glavin, V. A. Kochelap, T. L. Linnik, Phys. Rev. Lett. 96, 215504 (2006).

[58] O. Matsuda and O. B. Wright, Anal. Sci. 17, s216 (2000).

[59] A. A. Maradudin and D. L. Mills, Phys. Rev. B 11, 1392 (1975).

[60] M. Tomoda, O. Matsuda, O. B. Wright, and R. Li Voti, Appl. Phys. Lett. 90, 041114 (2007).

[61] D. Mounier, P. Picart, P. Babilotte, P. Ruello, J.-M. Breteau, T. Pézeril, G. Vaudel, M. Kouyaté, and V. Gusev, Opt. Express 18, 6767 (2010).

[62] T. Pezeril, N. Chigarev, P. Ruello, S. Gougeon, D. Mounier, J.-M. Breteau, P. Picart, and V. Gusev, Phys. Rev. B 73, 132301 (2006).

[63] B. Audoin, H. Meri, and C. Rossignol, Phys. Rev. B 74, 214304 (2006).

[64] T. Pezeril, P. Ruello, S. Gougeon, N. Chigarev, D. Mounier, J.-M. Breteau, P. Picart, and V. Gusev, Phys. Rev. B 75, 174307 (2007).

[65] O. B. Wright, B. Perrin, O. Matsuda and V. E. Gusev, Phys. Rev. B 78, 024303 (2008).

[66] H.-Y. Hao and H. J. Maris, Phys. Rev. B 64, 064302 (2001).

[67] O. L. Muskens, A. V. Akimov, and J. I. Dijkhuis, Phys. Rev. Lett. 92, 035503 (2004).

[68] B. C. Daly, T. B. Norris, J. Chen, and J. B. Khurgin, Phys. Rev. B 70, 214307 (2004).

[69] W. Singhsomroje and H. J. Maris, Phys. Rev. B 69, 174303 (2004).

[70] P. J. S. van Capel and J. I. Dijkhuis, Appl. Phys. Lett. 88, 151910 (2006). 